Published in "Neurobiology of Aging 76(): 80-95, 2019"

which should be cited to refer to this work.

\title{
Progressive signaling changes in the olfactory nerve of patients with Alzheimer's disease
}

\author{
Praveen Bathini ${ }^{\mathrm{a}}$, Antoine Mottas ${ }^{\mathrm{a}}$, Muriel Jaquet ${ }^{\mathrm{b}, 1}$, Emanuele Brai $^{\mathrm{c}}$, \\ Lavinia Alberi ${ }^{\mathrm{a}, \mathrm{b}, *}$ \\ a Department of Medicine, University of Fribourg, Fribourg, Switzerland \\ ${ }^{\mathrm{b}}$ Department of Neurology Research, Swiss Integrative Center for Human Health, Fribourg, Switzerland \\ ${ }^{\mathrm{c}}$ Neuro-Bio Ltd, Oxford, UK
}

Keywords:

Alzheimer's disease

Tau

Jagged 1

Reelin

Corpora amylacea

Olfaction declines with aging and appears to be a prodromal sign of cognitive decline in progressive neurodegenerative diseases. Nevertheless, very little is known about the pathophysiological changes underlying smell loss that may reflect early network dysfunction. A cross-sectional histoanatomical study was conducted on postmortem olfactory nerves of patients with increasing severity of dementia from mild cognitive impairment (MCI) to moderate and severe Alzheimer's disease. The olfactory bulbs and tracts show a prominent and progressive tauopathy in contrast to a weaker amyloid pathology localized to the glomerular region. Topological analysis of Notch signaling components reveals a transient increase in Jagged 1 expression in mitral cells of the olfactory bulb of patients with MCI and a gradual decline onwards. Analysis of the olfactory tract reveals an abundance of corpora amylacea, which declines starting from the MCI stage. With the increasing severity of dementia, corpora amylacea are characterized by a gradual shift in cytoskeletal proteins, tau, MAP2 and glial fibrillary acid protein, as well as by a decrease in their Reelin and Jagged 1 content. Our research indicates that the olfactory nerve undergoes early and sequential morphological and signaling alterations that correlate with the development of dementia suggesting that this structure may capture and propagate neuronal network imbalances to connected higher brain centers of the entorhinal cortex and hippocampus.

\section{Introduction}

Olfactory impairment is one of the common early signs in progressive neurodegenerative diseases (ND) such as Alzheimer's disease (AD), Parkinson's disease (PD), Down syndrome, Frontotemporal dementia (FTD), Huntington's disease, and amyotrophic lateral sclerosis (Barrios et al., 2007; Brai and Alberi, 2018; Doty and Kamath, 2014; Hüttenbrink et al., 2013; Mesholam et al., 1998). In humans, the olfactory system is composed of a primary olfactory area, constituted by the olfactory sensory epithelium lining the upper nasal cavity (Fig. 1A), and central olfactory areas composed of the olfactory bulb (OB) and the olfactory nerve connected to the limbic cortices and nuclei of the basal forebrain and midbrain. Besides the eye, the olfactory network is devoid of a blood-brain barrier making it a susceptible port of entry for xenobiotics but also an ideal anatomical window for diagnostic and

\footnotetext{
* Corresponding author at: Swiss Integrative Center for Human Health, Passage du Cardinal, 13B, 1700 Fribourg, Switzerland. Tel.: +41-26-3006512; fax: +41-263006510 .

E-mail address: lavinia.alberi@unifr.ch (L. Alberi).

1 Department of BioMedical Research, University of Bern, Bern, Switzerland.
}

therapeutic applications (Brai and Alberi, 2018; Hock et al., 1998; Sattler et al., 2011). Furthermore, early imbalances in cholinergic, serotoninergic, and noradrenergic transmission may translate into smell deficits based on the corticofugal monoaminergic fibers innervating the olfactory nerve (Arendt et al., 1983; Kovacs et al., 2003).

In support of the early olfactory phenotype in dementia, studies using mouse models of AD report an olfactory deficit (Wesson et al., 2010; Yao et al., 2017; Yoo et al., 2017). Furthermore, loss of function models for signaling pathways implicated in AD (Brai et al., 2016; Chin et al., 2007; Cuchillo-Ibañez et al., 2016) show an olfactory impairment associated with spatial memory loss (Notch1cKO, Jagged1cKO, and Reeler) (Brai and Alberi, 2015, 2018; Brai et al., 2014; Larson et al., 2003). Both Notch and Reelin signaling play an important role in neural development and support synaptic plasticity in the adult brain (Alberi et al., 2013; Herz and Chen, 2006). Jagged1 and Reelin are ligands for Notch and ApoER2 transmembrane receptors, respectively. On ligand-binding, Notch and Reelin signaling functionally interact to increase synaptic potentiation (Brai et al., 2015) and can be viewed as part of a common signalosome shaping neuronal networks and enhancing 
connectivity. Interestingly, Reelin has been observed in extracellular structures, called corpora amylacea (CAm) (Notter and Knuesel, 2013). These varicosities are glycoprotein-based polyglucosan bodies typically formed in the aging brain (Rohn, 2015) in response to senescence and stress (Martin et al., 1991; Rohn, 2015). CAm are found either in the brain parenchyma, ependyma, or perivascular space and they have been shown to increase in number in the majority of NDs (AD, PD, Huntigton's disease, multiple sclerosis, and epilepsy) (Pirici and Margaritescu, 2014). Their origin and function remains a matter of debate, but recent proteomic data confirm the coexistence of neuronal and glial proteins in addition to microbial species in CAm, supporting their role as a waste disposal system (Pisa et al., 2018). As their content and density changes with aging and ND, they may offer insights into the biological repertoire of brain cells in the diseased versus healthy state. Nevertheless, to date, no studies have reported stage-specific changes of signaling molecules in CAm with the progression of dementia.

This study consists of a cross-sectional histoanatomical analysis of the olfactory nerves from patients with $\mathrm{MCI}$, moderate and severe $\mathrm{AD}$, as well as healthy controls. We confirm a prominent
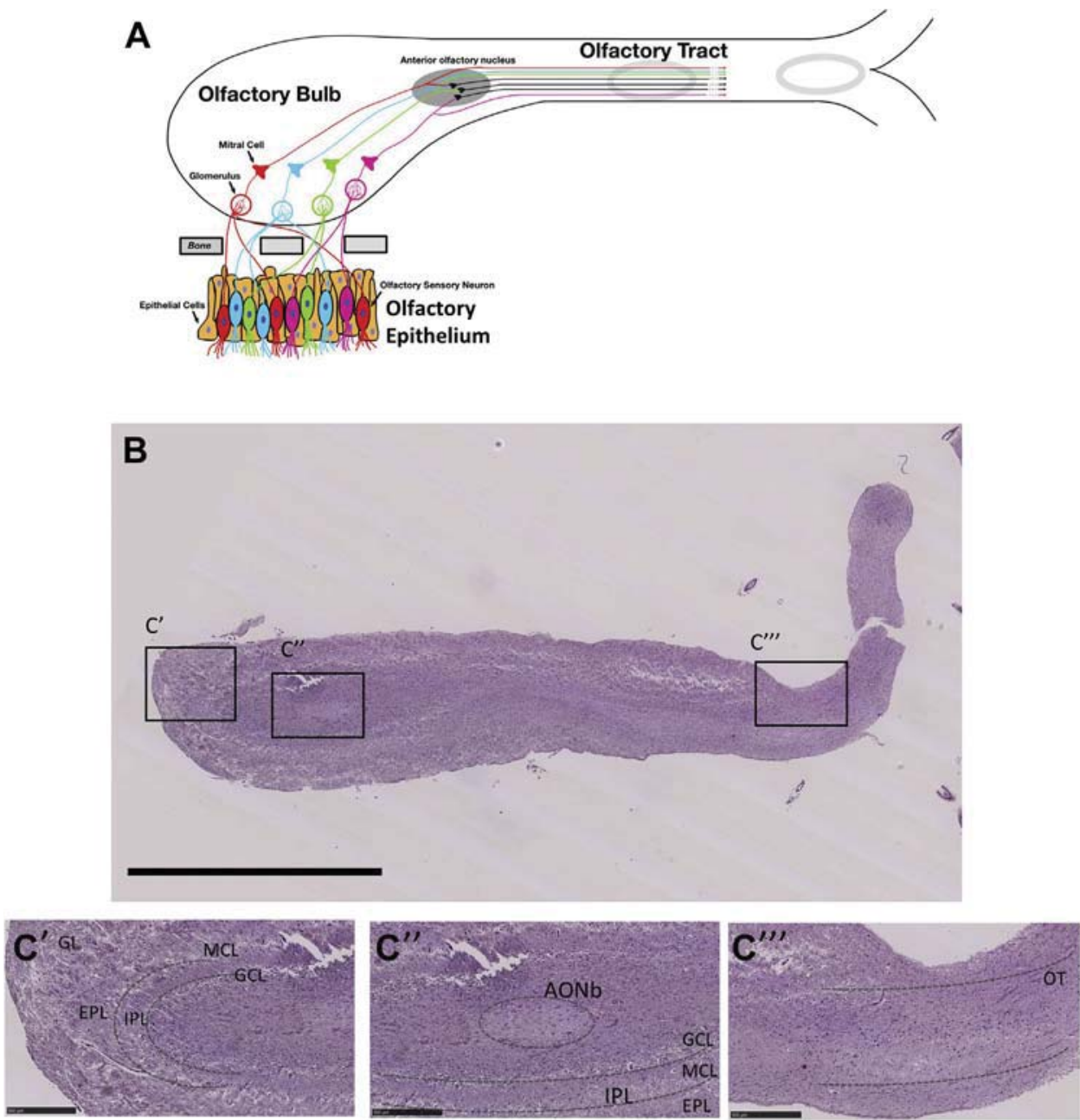

D

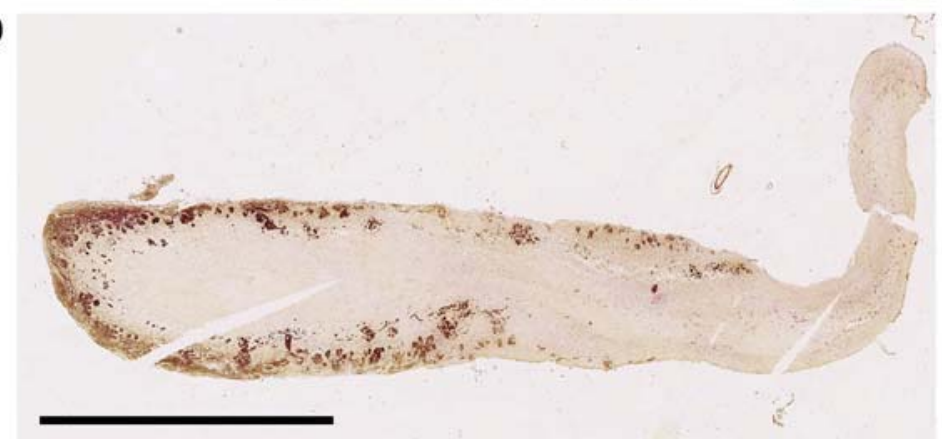

Fig. 1. Olfactory nerve anatomy and regions of interest examined in the study. (A) Drawing of the human olfactory system including the olfactory epithelium, the olfactory bulb, and the olfactory tract (B) Representative Hematoxylin and Eosin staining of the human olfactory nerve with boxed regions indicating the region of interests analyzed in this study and magnified below. ( $\left.\mathrm{C}^{\prime}\right)$ Olfactory bulb with visible substructures, the glomerular region (GL), the external plexiform layer (EPL), the mitral cell layer (MCL), the internal plexiform layer (IPL), and the granular cell layer (GCL). ( $C^{\prime \prime}$ ) Magnification of the bulbar anterior olfactory nucleus (AONb) and underlying structures. ( $\mathrm{C}^{\prime \prime \prime}$ ) The olfactory tract (OT) with typical axonal stria and CAm. (D) Chromogen immunohistochemistry for OMP, localizing the GL region in the olfactory nerve. Scale bar in B and D is 2 mm and in the inserts in $\mathrm{C}^{\prime}-\mathrm{C}^{\prime \prime}$ are $500 \mu \mathrm{m}$. Abbreviations: GL, glomerular region; OMP, olfactory marker protein and CA, corpora amylacea. 
tauopathy in the $\mathrm{OB}$, olfactory tract (OT), and anterior olfactory nucleus (AON) accompanied by transient astrogliosis. Furthermore, we observe specific alterations in the Notch ligand, Jagged1, in mitral cells of the OB. Finally, we track the density of CAm in the OT and their changes in Jagged 1 and Reelin content and cytoskeletal markers. This study offers the first evidence of specific switches in signaling modulators, along with structural and pathological events in the olfactory nerves of progressively demented patients.

\section{Materials and methods}

\subsection{Human tissue}

The human brain tissue samples were generously provided by the Medical Research Council Brain Bank for Dementia Research, Oxford, UK. We received paraffin-embedded horizontally sectioned olfactory nerves from patients diagnosed with increasing severity of dementia according to the minimal mental score evaluation at the last visit, the postmortem pathological evaluation based on the Braak staging and Consortium to Establish a Registry for Alzheimer's Disease (Table 1 and Supplementary Table 1). The research was approved by the ethical committee of Clinical Research of the Canton Vaud (CER-VD, n. 2016-01627) Medical Research Council Brain Bank for Dementia Research, Oxford, UK (OBB485).

\subsection{Antibodies and labeling reagents}

The primary antibodies used for the chromogen immunohistochemistry on olfactory nerves' sections were rabbit anti- $\beta$-amyloid-42 (A $\beta-42 ; 1: 250$; D54D2 XP, Cell Signaling, USA), rabbit antiphosphorylated tau (p-tau; phosphorylated T205) (1:500; ab4841; Abcam, UK), mouse anti-olfactory marker protein (OMP; 1:500; sc365818, Santa Cruz Biotechnology, USA), mouse anti-glutamate decarboxylase-67 (GAD-67; 1:250; sc-28376, Santa Cruz Biotechnology, USA), rabbit anti-Jagged1 (1:500; ab7771, Abcam, UK), and mouse anti-Jagged1 (1:250; H00000182-M01A, Novus Biologicals, UK). Single chromogen immunohistochemistry for p-tau, $A \beta-42$, and Jagged1 was performed using the biotinylated donkey antirabbit (cat.no. 711-065-152, Jackson Immunoresearch Europe Ltd, UK) and VECTOR Red Alkaline Phosphatase Substrate Kit (SK-5100; Vector Laboratories, US). Single chromogen immunolabeling for OMP was performed as previously described using biotinylated donkey anti-mouse (cat. no. 715-065-150, Jackson Immunoresearch Europe Ltd, UK) and 3,3'-Diaminobenzidine (D12384, SigmaAldrich, USA). Single chromogen immunolabeling for mouse antiJagged1 was performed using the emerald green reagent from the Double Stain IHC Kit (ab183285, Abcam, UK) following the manufacturer's instructions. For double chromogen immunolabeling of rabbit anti-Jagged 1 and mouse anti-GAD67, we used the complete Double Stain IHC Kit (ab183285, Abcam, UK). The primary antibodies for the immunofluorescence were polyclonal goat antiNotch1 against the c-terminus (1:500; sc-6014, Santa Cruz Biotechnology, USA), goat anti-Notch1 extracellular portion (1:500; sc-23299, Santa Cruz Biotechnology, USA), rabbit anti-Jagged1

Table 1

Summary of cases per condition

\begin{tabular}{lrlllcll}
\hline Staging & N & Age & F:M & Braak & $\begin{array}{c}\text { MMSE at } \\
\text { last visit }\end{array}$ & PM delay (h) & CSF (pH) \\
\hline MCI & 9 & $89 \pm 1.6$ & $7: 2$ & $2.6 \pm 0.2$ & $26.4 \pm 0.8$ & $45.8 \pm 11.0$ & $6.2 \pm 0.1$ \\
Moderate & 8 & $89 \pm 1.3$ & $3: 5$ & $4.0 \pm 0.0$ & $16.3 \pm 2.5$ & $44.9 \pm 6.9$ & $6.2 \pm 0.8$ \\
Severe & 11 & $81 \pm 3.2$ & $7: 4$ & $5.6 \pm 0.2$ & $0.0 \pm 0.0$ & $43.3 \pm 8.1$ & $6.2 \pm 0.6$ \\
Controls & 10 & $77 \pm 2.5$ & $5: 6$ & $0.8 \pm 0.1$ & N/A & $41.9 \pm 6.9$ & $7.3 \pm 0.6$ \\
\hline
\end{tabular}

Key: CSF, cerebrospinal fluid; F, female; M, male; MMSE, minimal mental score evaluation; MCI, mild cognitive impairment; PM, postmortem.
(1:500; ab7771, Abcam, UK), mouse anti-Reelin (1:500; ab78540, Abcam, UK), rabbit anti-Tbr2 (1:500; ab23345, Abcam, UK), rabbit anti-glial fibrillary protein (GFAP; 1:5000, IS52430, Dako, US), mouse anti-Ubiquitin (1:500; sc-8017, Santa Cruz Biotechnology, USA), Chicken anti-MAP2 (1:1000; NB300-213, Novus Biologicals, UK), rabbit anti-synaptophysin (1:500; ab14692, Abcam, UK), mouse anti-PSD95 (1:500; sc-32290, Santa Cruz Biotechnology, USA), rabbit anti-p-tau (phospho T205) (1:500; ab4841; Abcam, UK), mouse anti-NF200 (1:500; cat. no. 1178709; Boehringer Mannheim Biochimica, Germany), goat anti-tau $(1.25 \mu \mathrm{g} / \mathrm{mL}$; AF3494, R\&D systems, USA). Misfolded $\beta$-sheets were detected using Thioflavin S (100 mM; CAS 1326-12-1, Santa Cruz Biotechnology, USA). The secondary antibodies used for the immunofluorescence were Cy3 donkey anti-goat (cat. no. 705-165-147), Сy3 donkey anti-mouse (cat. no. 715-165-150), Cy5 donkey anti-mouse (cat. no. 715-605-150), Cy5 donkey anti-chicken (cat. no. 703-175155), Cy2 donkey anti-rabbit (cat. no. 711-545-152), Cy2 donkey anti-mouse (cat. no. 715-225-150) and Cy2 donkey anti-goat (cat. no. 705-225-147). All fluorescently conjugated antibodies were purchased from Jackson Immunoresearch Europe Ltd and were all diluted 1:1000. Hematoxylin-Eosin (H\&E) staining was performed using a commercial kit (H\&E fast staining kit, cat. no. 9194, Roth, Germany).

\subsection{Immunohistochemistry}

Chromogen immunohistochemistry to detect the expression of Jagged1, GAD67, OMP, A $\beta-42$, and p-tau was done using Fast Red, Emerald green, and 3,3'-Diaminobenzidine substrates. The remaining immunostainings were performed using directly conjugated fluorescent secondary antibodies. For all immunolabeling, deparaffinization, antigen retrieval, and incubation with primary antibodies were conducted as follows: (1) deparaffinization step: human sections were deparaffinized in Xylol $(3 \times 10$ minutes $)$ and rehydrated in decreasing concentrations of ethanol $(2 \times 100 \%, 2 \times$ $96 \%, 1 \times 80 \%, 1 \times 70 \%$ and $2 \times$ distilled water for 5 minutes each); (2) antigen retrieval was performed by warming the sections with $10 \mathrm{mM}$ of sodium citrate buffer ( $\mathrm{pH} 6)$, for 45 minutes at $65^{\circ} \mathrm{C}$ in a water bath. Thereafter, sections were washed 3 for 5 minutes with Trizma-based salt solution (TBS) and further blocked for 1 hour at room temperature with a blocking solution (TBS containing $10 \%$ fetal bovine serum and $0.1 \%$ Triton). Only for the chromogenstained sections, quenching of the peroxidase using 3\% $\mathrm{H} 2 \mathrm{O} 2$ was performed before antigen retrieval; (3) incubation with primary antibodies was conducted at the aforementioned concentrations in TBS with $1 \%$ fetal bovine serum and $0.1 \%$ Triton overnight at $4{ }^{\circ} \mathrm{C}$. The next day, for the chromogen stainings, after washing 3 times for 5 minutes, the sections were incubated with either Horseradish peroxidase-polymer anti-mouse (Emerald Green), AP-polymer anti-rabbit (Fast Red) following the manufacturer's instructions (Double IHC kit, ab183285) or with biotinylated donkey anti-mouse followed by Horseradish peroxidase streptavidin as previously described (Brai et al., 2016). For the fluorescent immunolabeling, directly conjugated secondary antibodies were incubated for 3 hours at room temperature, 3 washes $\times 5$ minutes with TBS followed. In one staining (NF200 and Notch1), sections were incubated with Thioflavin S, followed by $2 \times 5$ minutes washes with distilled water. All fluorescent immunolabeled sections were incubated for 10 minutes with 4',6-diamidino-2-phenylindole (Cat. n. 10236276001 Roche, Switzerland) and washed for $2 \times 5$ minutes with TBS. To eliminate the autofluorescence caused by lipofuscin accumulation in aged cells, sections were dipped in Sudan Black (Sudan Black B; 199664 Sigma-Aldrich, USA) for 5 minutes and destained from excess particles using $70 \%$ clean ethanol. Thereafter, the sections were washed $2 \times 5$ minutes with TBS and mounted 
with custom-made aqueous mounting media containing 1,4Diazabicyclo[2.2.2] octane (8.03456 EMD Millipore, USA). For the chromogen sections, dehydration was followed by mounting with organic media (DPX, 06522, Sigma Aldrich, USA).

\subsection{Imaging quantification}

Chromogen-immunolabeled sections were imaged using a slide scanner (NanoZoomer Hamamatsu 2.0 HT, Japan) with a $40 \times$ objective, whereas fluorescent-immunolabeled sections were imaged using a Confocal microscope (Zeiss LSM 800) at $40 \times$ oil immersion objective with $\mathrm{Z}$ intervals of $1 \mu \mathrm{m}$. The density of CAm was performed on the H\&E stain of the medial OT using QuPath (https://qupath.github.io/) and a custom-made script to allow for unbiased counting based on a watershed detection and signal intensity (Supplementary Fig. 6A and $\mathrm{A}^{\prime}$ ). An average of $500 \mathrm{CAm} /$ patient was counted (Supplementary Fig. 6B) and analyzed for area/diameter (Supplementary Fig. 6C). A $\beta$ 42 and p-tau immunostainings were analyzed on randomly selected regions of interest (ROIs) on the glomeruli (GL), OT, and AON (5 ROIs/ segment/section) using the particle analyzer tool of the Image (https://imagej.nih.gov/ij/). Integrated fluorescence densities of Jagged-1, Reelin, GFAP, MAP2, and tau were measured with ImageJ using the oval tool to draw an ROI on the analyzed object (mitral cell or CAm) and adding it to the ImageJ ROI manager. For each stage, a minimum of 100 mitral cells and 200 CAm were counted and analyzed. An integrated signal density of Jagged1 expression in the CAm was performed on randomly selected ROIs from the NDP.view2 software (Hamamatsu, Japan) (Supplementary Fig. 6D). Imported 2D images in Image (Supplementary Fig. 6D') were processed using the ImageJ Trainable Weka segmentation v3.3.28 plugin. RGB images were deconvoluted and CAm with diameter $>10 \mu \mathrm{m}$ were analyzed (Supplementary Fig. 6E). The relative amount of chromogen Jagged1 per CAm on an average of $200 \mathrm{CAm} /$ staging was assessed (Supplementary Fig. 6F). Colocalization analysis of fluorescent puncta was conducted on 60 randomly selected CAm/staging using the colocalization plugin tool of Imagej. Mander's correlation coefficient was used to assay the extent of pixel overlap as previously described (Brai et al., 2015, 2016).

\subsection{Statistical analysis}

Normality of variance was assessed for all biomarkers within each group using the Shapiro-Wilk Test. Paired comparison were made between subsequent stages of dementia, healthy controls versus $\mathrm{MCl}, \mathrm{MCl}$ versus moderate $\mathrm{AD}$, and moderate $A D$ versus severe $A D$ to assess any progressive change with the increasing severity of dementia. For paired comparison between normally distributed samples, Student's t-test with unequal variances was used; otherwise, for non-normal distributed data, Mann Whitney $U$-Test and Wilcoxon signed ranks test were used. Frequency distribution was analyzed using the Kolmogorov-Smirnov (KS) test. The $\alpha$ significance level was considered 0.05 with a $p$-value said to be significant when being $p<$ $\alpha$. $\alpha$ values were corrected for the type I error, by dividing the $\alpha$ value $=0.05$ to the number of subjects. Correlations were conducted according to the Pearson's method. All statistical analyses were performed using the Excel Plugin Real statistics from Charles Zaiontz (http://www.real-statistics.com/).

\section{Results}

\subsection{Progressive proteinopathy with the severity of dementia}

We obtained olfactory nerves from patients diagnosed with $\mathrm{MCI}$, moderate $\mathrm{AD}$, severe $\mathrm{AD}$, and healthy controls (Table 1 and Supplementary Table 1). Patients were of both genders, age- matched, scored based on the Consortium to Establish a Registry for Alzheimer's Disease evaluation, and staged according to the Braak criteria (Braak et al., 1993, 2006), whereas the minimal mental score was only reported from the last premortem visit in the patients only (Supplementary Table 1). We first performed an $\mathrm{H} \& \mathrm{E}$ staining on horizontal sections to identify the olfactory regions and their substructures (Fig. 1B). Of 38 specimens, all presented the OT, $76 \%$ the $\mathrm{OB}$, and $53 \%$ the bulbar $\mathrm{AON}$ (Fig. 1C-C"). The position of the OB was further confirmed using immunohistochemistry for the OMP, which labels the axons of the olfactory sensory neurons (OSN) innervating the GL (Fig. 1D). We next conducted a positional analysis of insoluble aggregates by using immunohistochemistry for phosphorylated tau protein (p-tau) (Fig. 2) and $A \beta-42$ (Fig. 3). We observe a progressive increase in p-tau neuropils and tangles in all aforementioned regions already evident at the MCI stage (Fig. 2A' and $A^{\prime \prime}$ and Supplementary Fig. 2A). Overall, the GL region shows fewer tau tangles as compared to the AON and OT, and p-tau depositions appear in neuropil form (Fig. 2A and Supplementary Fig. 2A, GL). In the severe stage, among visible neurofibrillary tangles (NFTs) (Fig. 2A' and $\mathrm{A}^{\prime \prime}$, black arrows), p-tau fibrils reveal the meshed network of the AON and the parallel mitral axons bundles spanning the OT (Fig. 2A' and $A^{\prime \prime}$ and Supplementary Fig. 2A). The quantification of the chromogen signal indicates a significant rise in p-tau particles in patients with $\mathrm{MCI}$ as compared with healthy controls in the $\mathrm{GL}(\mathrm{r}=0.58, p=0.0005), \mathrm{AON}(\mathrm{r}=0.61, p=0.002)$, and OT $(r=0.46, p=0.001)$. In the moderate condition, the accumulation in the explored regions was comparable to $\mathrm{MCI}$. With the progression to the severe stage, there is a growing tauopathy in the GL, a close to significant rise in the OT regions (GL: $\mathrm{r}=0.63, p<0.001$; OT: $\mathrm{r}=0.28, p=0.05$ ) but only an increasing trend in the AON $(r=0.31, p=0.13)$. Despite the plateauing effect between the $\mathrm{MCI}$ and moderate stage, the p-tau load correlates positively with the Braak staging (Braak et al., 1996) (GL: $\mathrm{R}=0.55, p<0.001$; AON: $\mathrm{R}=0.69, p<0.001$; OT: $\mathrm{R}=0.60, p<0.001)$. Based on our previous studies indicating the presence of Notch1 in fibrillary aggregates in the hippocampus and cortex of patients with severe AD (Brai et al., 2016), we investigated the localization of Notch1 in p-tau, NF200, and Thioflavin-S-positive fibrils. We observe that in neurons of mitral cell layer, the AON and OT of severe AD specimen, all tangled neurons are also Notch1-positive (Supplementary Fig. $1 \mathrm{~A}-\mathrm{C}$ ). To further characterize the progressive neuropathology, we explored the incidence of $A \beta$ species expression in the aforementioned areas. $A \beta-42$ puncta are visible as early as in $\mathrm{MCI}$ in the AON (Fig. 3A'), they appear in the GL at the moderate stage (Fig. 3A) but are sparsely present in the OT over the course of the disease (Fig. $3 \mathrm{~A}^{\prime \prime}$ ). From the moderate stage on, visibly diffused plaques are detectable in the GL and AON regions, clearly distinguishable from the core plaques present in the cortex (Supplementary Fig. 2B). The morphology of the amyloid plaques suggests that the $A \beta$ species may be intracellular conferring its diffuse pattern (Gouras et al., 2005). The counting of the $A \beta$ 42-positive particles reveals that there is a significant accumulation of $A \beta$ in the GL at the moderate stage $(r=0.46, p=0.04)$, which increases further in severe patients $(r=0.63, p=0.002)$ and positively correlates with the Braak staging $(\mathrm{R}=0.65, p<$ 0.001 ) (Fig. 3B). In the AON, we observe a large variability in $A \beta$ accumulation at all stages. Nevertheless, the trend is positive, and there is a significant rise in the cross-sectional comparison between moderate or severe AD and healthy controls $(\mathrm{r}=0.78, p<$ $0.001 ; r=0.5, p=0.04$ ) (Fig. 3C). On the other hand, in the OT, despite an initial increase at $\mathrm{MCI}(\mathrm{r}=0.4, p=0.03)$, the amyloid pathology plateaus afterward (Fig. 3D). To further assess whether the amyloidogenesis in the GL region could result from the 
A
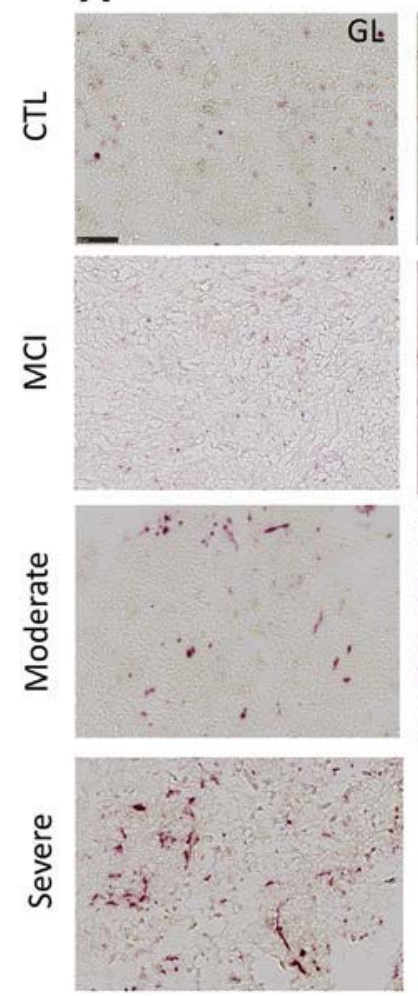

$A^{\prime}$
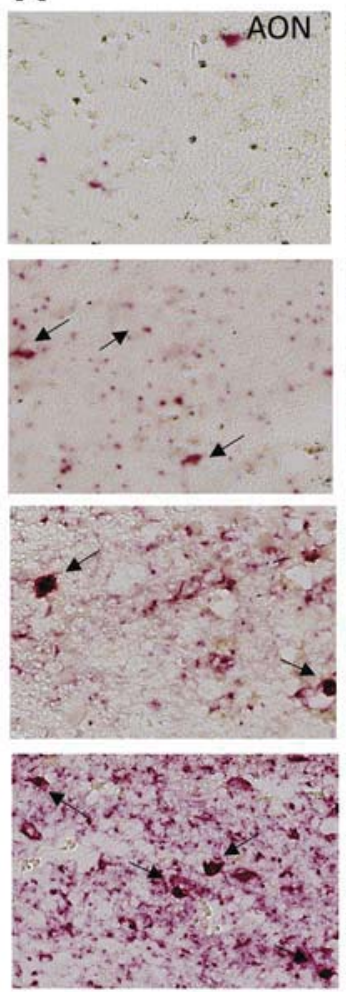

A"
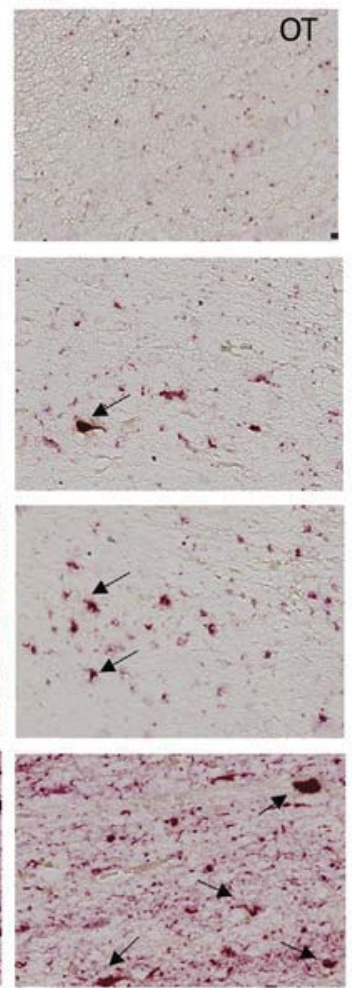

B

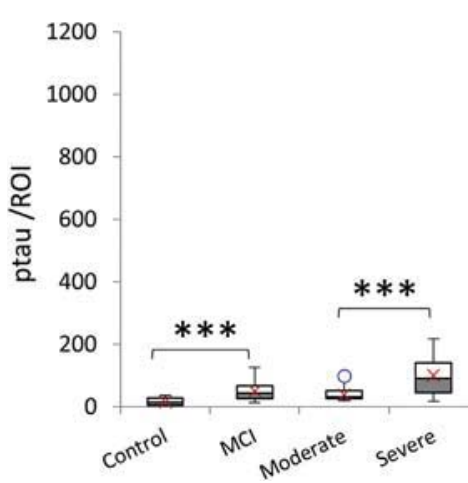

C

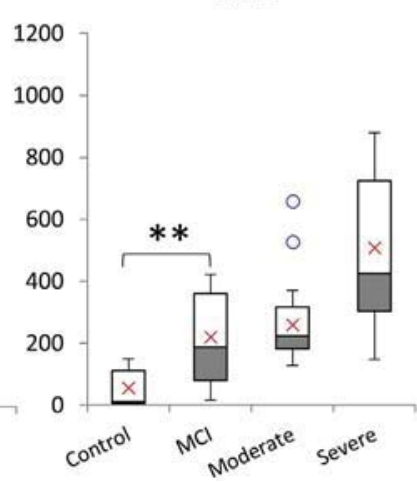

D

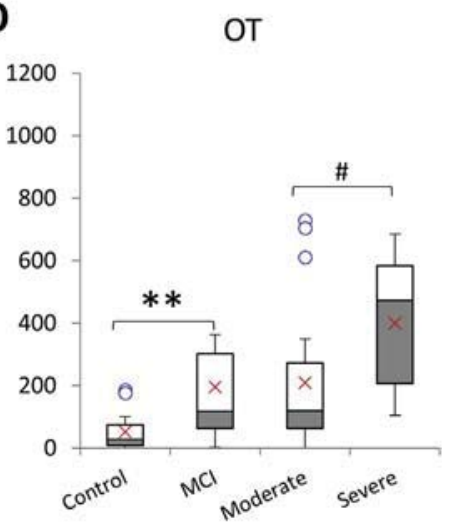

Fig. 2. Progressive tau pathology in the olfactory nerve with the increasing severity of dementia. (A- $A^{\prime \prime}$ ) Representative images of p-tau chromogen immunolabeling in the glomerular region (GL), the anterior olfactory nucleus (AON), and the olfactory tract (OT) of the olfactory nerve. (A) The GL shows an increase in scattered p-tau puncta in MCI as compared to healthy controls. In moderate AD, p-tau labels neuropil structures, which increase in the severe stage. $\left(A^{\prime}\right)$ Positive p-tau puncta are visible in the AON already in healthy controls and p-tau aggregates increase progressively from $\mathrm{MCl}$ to moderate, showing a spread tau pathology with visible tangles (black arrows), which gain in intensity and frequency in the severe patients. ( $\mathrm{A}^{\prime \prime}$ ) In the OT, p-tau puncta are dispersed in the healthy controls and take a neuropil shape in MCI, with scattered NFTs (black arrows). The density of the p-tau aggregates progressively increases in moderate patients and p-tau-labeled neuropils decorating the olfactory axon bundles. Box plots summarizing the quantification of the p-tau particles in the progressive stages of dementia (B) in the $G L(C)$ in the AON, and (D) in the OT. On the box plot, blue empty dots are maximum and minimum outliers, and red crosses represent the mean value of the sampled data. $\#=p: 0.05,{ }^{*}=p<0.05,{ }^{* *}=p<0.01$, and ${ }^{* * *}=p<0.001$. Scale bar in A is $50 \mu \mathrm{m}$ and applies to all panels. Abbreviations: $\mathrm{AU}$, arbitrary units; p-tau, phosphorylated tau; ROI, region of interest. (For interpretation of the references to color in this figure legend, the reader is referred to the Web version of this article.)

innervation of the OSN, we conducted a fluorescent OMP staining. We observe that OMP expression in the GL from being finely punctuated in the controls and $\mathrm{MCI}$ starts to form brighter aggregates at the moderate stage (Supplementary Fig. 3A). Analysis of the fluorescence intensity shows that OMP increases significantly in the GL at the moderate stage as compared to MCI ( $\mathrm{r}=$ $0.51, p=0.001$ ) and stabilizes onward (Supplementary Fig. 3B).

To understand whether the proteinopathy observed in the OB and tract correlates with neuroinflammatory processes, we conducted GFAP immunolabeling in the GL and OT regions (Fig. 4A and B). From the quantification of fluorescence immunoreactivity, we observe a transient but not significant increase in GFAP immunoreactivity in the GL due to the variability of the GFAP intensities in the samples examined $(r=0.15, p=0.4)$ (Fig. $4 \mathrm{~A}$ and $\mathrm{C}$ ). On the other hand, in the OT, the reactive astrogliosis is more prominent and has its peak in the moderate $\mathrm{AD}(\mathrm{r}=0.56, p<$ 0.001 ) (Fig. 4B and D). At the severe stage, in both GL and OT, astrogliosis appears to resolve as compared to $\mathrm{MCI}$ and moderate stage, respectively (GL: $\mathrm{r}=0.61, p=0.0002$; OT: $\mathrm{r}=0.76, p<$ 0.001 ) (Fig. 4C and D). 
A
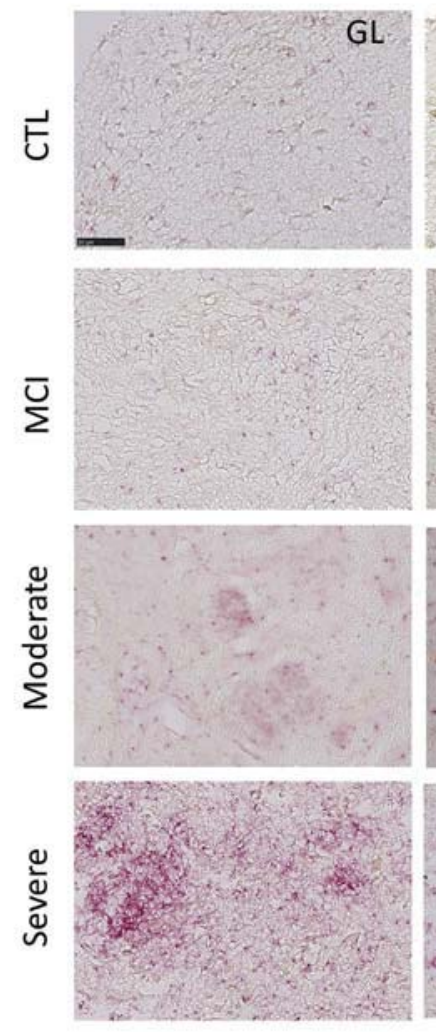

GL

B

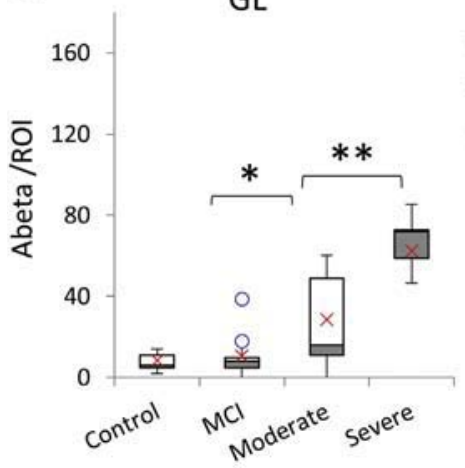

$A^{\prime}$
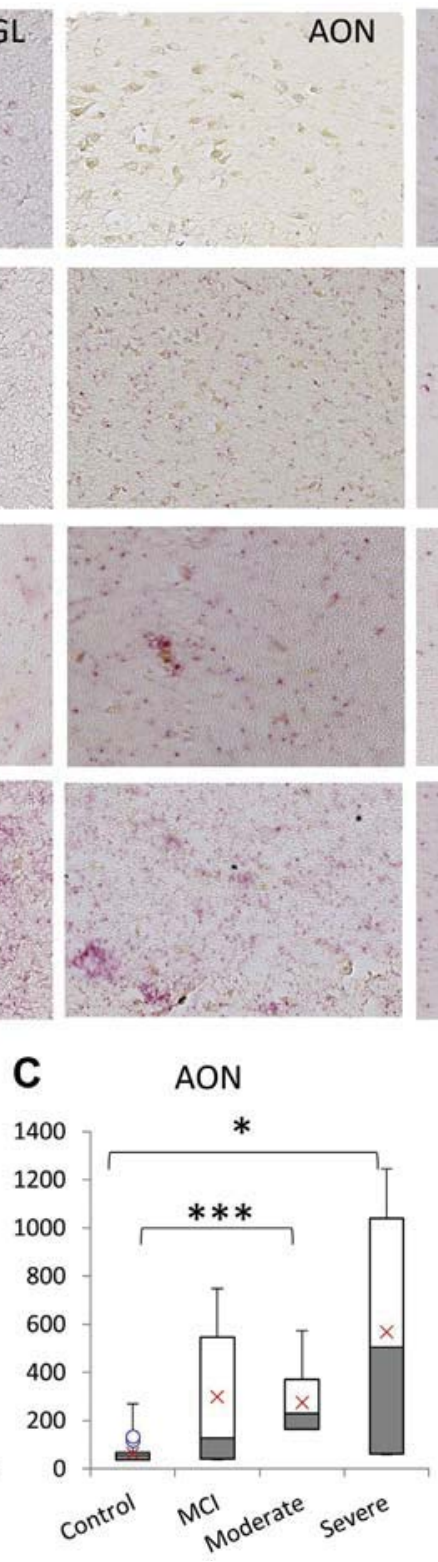

$\mathbf{A}^{\prime \prime}$
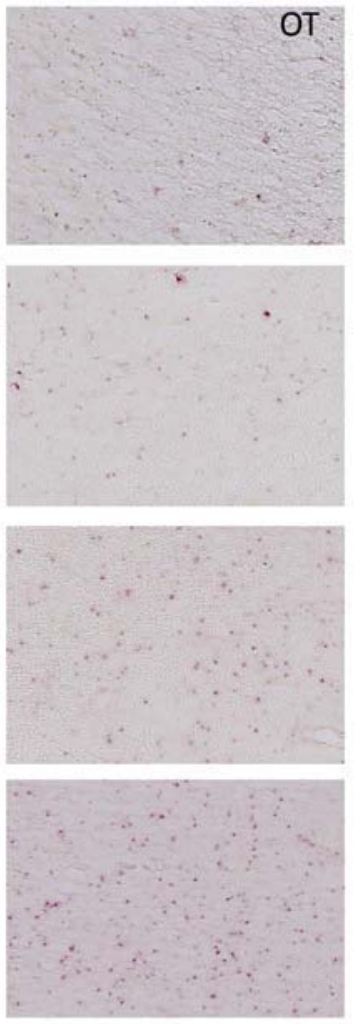

D $\quad$ OT

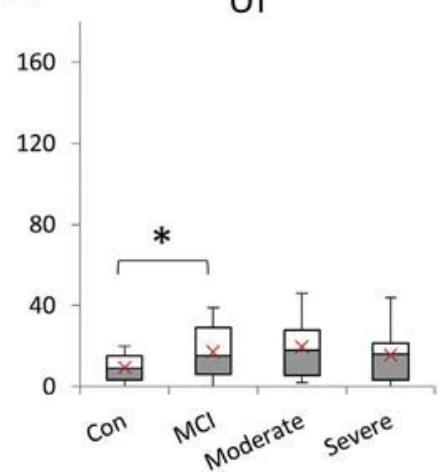

Fig. 3. Localized amyloid pathology in the olfactory nerve of demented patients. $\left.A-A^{\prime \prime}\right)$ Representative images of $A \beta-42$ chromogen immunolabeling in the glomerular region (GL), the anterior olfactory nucleus (AON), and the olfactory tract (OT) of the olfactory nerve. (A) In the GL, a diffuse A - 42 pathology is first apparent at the moderate stage and is more abundant in the severe condition, whereas $\mathrm{MCI}$ shows no $\mathrm{A} \beta-42$ deposition comparably to controls. $\left(\mathrm{A}^{\prime}\right)$ In the $\mathrm{AON}, \mathrm{A} \beta-42$ puncta are visible already in $\mathrm{MCI}$, and the magnitude of the amyloid aggregates increases in the subsequent moderate and severe stages. ( $A^{\prime \prime}$ ) In the OT, $A \beta-42$ particles are sparsely distributed. Box plots summarizing the quantification of the $\beta$-amyloid particles in the progressive stages of dementia (B) in the GL, (C) in the AON, and (D) in OT. On the box plot, blue empty dots are maximum and minimum outliers, and red crosses represent the mean value of the sampled data. ${ }^{*}=p<0.05,{ }^{* *}=p<0.01$, and ${ }^{* * *}=p<0.001$. Scale bars in A are $50 \mu \mathrm{m}$ and this applies to all panels. Abbreviations: AU, arbitrary units; ROI, region of interest. (For interpretation of the references to color in this figure legend, the reader is referred to the Web version of this article.)

\subsection{Jagged 1 and Notch 1 expression in human mitral cells}

To understand whether the proteinopathy occurring in the olfactory network is reflected in Notch signaling changes, we investigated the expression pattern of Notch 1 and Jagged 1 in mitral cells. This neuronal population represents the principal neurons of the $\mathrm{OB}$ receiving direct input from the OSN and transferring the impulse to the AON and entorhinal and piriform cortices (Brai and Alberi, 2018). We distinguished mitral cells based on their topological position respective to the GL and the external plexiform layer, the larger size of their nuclei $\left(50 \mu \mathrm{m}^{2}\right)$ and the pyramidal shape of their soma (Fig. 5A, arrows).
Fluorescent immunohistochemistry for Notch1 and Jagged1 revealed that mitral cells, identified relative to OMPimmunolabeled GL, expressed fluctuating levels of Jagged1, in contrast to a more stable Notch1 expression (Fig. 5A', inserts). Quantification of the fluorescence intensities of Jagged1 and Notch1 in 100 mitral cells per condition indicates that Jagged1 expression is transiently elevated in $\mathrm{MCI}(\mathrm{r}=0.38, p=0.001)$ and decreases afterward $(r=0.53, p=0.002)$ (Fig. 5B). Surprisingly, Notch1 expression in mitral cells remained unchanged over the course of the disease (Fig. 5C), suggesting that Jagged 1 may be the relevant signaling modulator in human mitral cells. To confirm the identity of mitral cells, double immunolabeling for Tbr2 and 

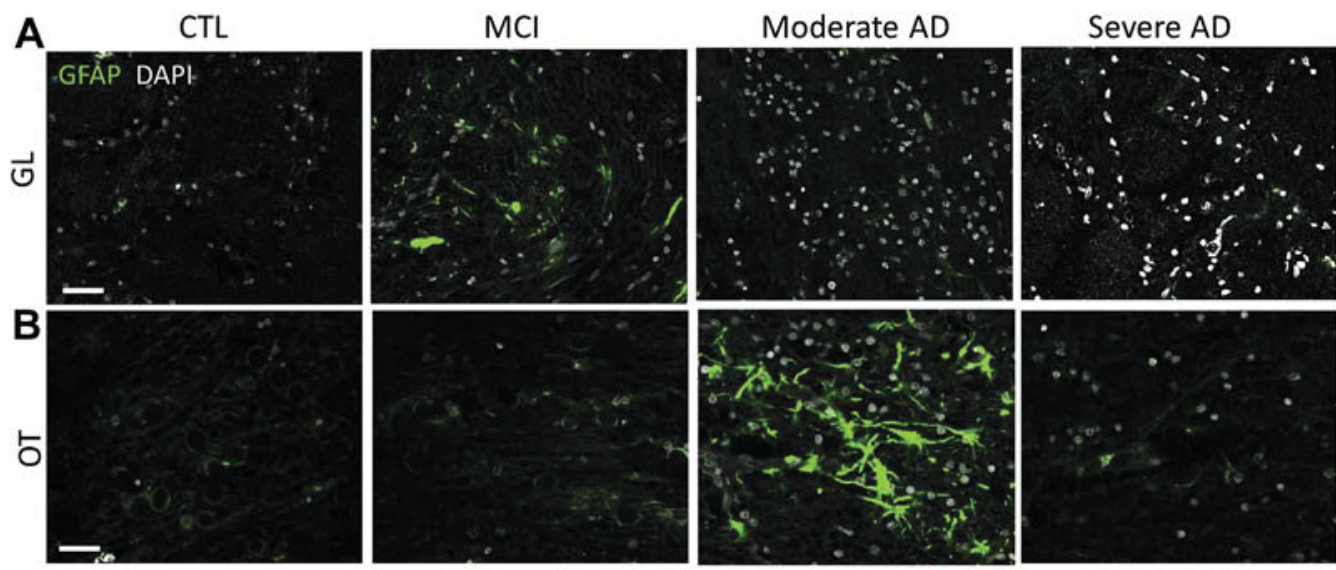

C
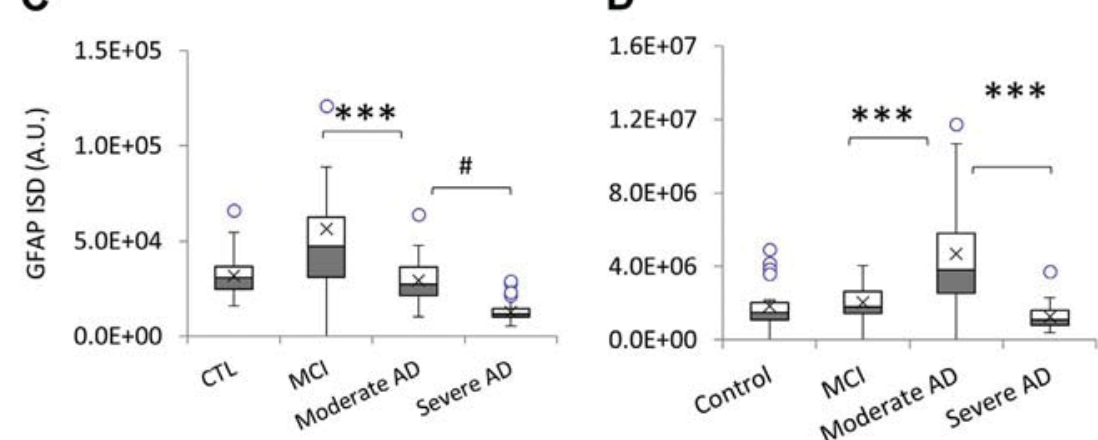

Fig. 4. Timing of astrogliosis in OT and GL. Representative panels of single GFAP immunolabeling in GL (A) showing a progressive decline in astrogliotic reaction from MCI to severe $\mathrm{AD}$ and $(\mathrm{B})$ in the OT abundant astrogliosis at the moderate AD stage. Box plots summarizing the ISD analysis of GFAP in the (C) GL and (D) OT over the progression of dementia. On the box plot, blue empty dots are maximum and minimum outliers and black crosses represent the mean value of the sampled data. Scale bars in $\mathrm{A}$ and B are $50 \mu \mathrm{m}$. \# $=p: 0.07^{* * *}=$ $p<0.001$. Abbreviations: GL, glomerular region; OT, olfactory tract; GFAP, glial fibrillary acid protein; ISD, integrated signal density. (For interpretation of the references to color in this figure legend, the reader is referred to the Web version of this article.)

Jagged1 shows that all Tbr2-positive cells express high levels of Jagged1 (Fig. 5D, white arrows).

\subsection{Neuronal identity of corpora amylacea in the OT}

Using H\&E staining, we observe large rounded extracellular vesicles, which were hemalum-impregnated with a diameter of $>10 \mu \mathrm{m}$, resembling CAm (Fig. 6A and Supplementary Fig. 4A). Sudan Black staining, which labels the myelinated axons of the OT (Ineichen et al., 2017), shows that CAm are devoid of impregnation in their core, excluding any oligodendrocyte-derived content (Supplementary Fig. 4B). The composition and origin of CAm has been long debated, and a recent paper demonstrated that CAm contain neoetitopes recognized by IgM traces present in primary and secondary antibodies arguing that CAm are neither neuronal nor glial-derived (Augé et al., 2017). A later, proteomic analysis has confirmed that CAm extracted from the insular cortex of patients with $A D$ and controls contain neuronal and glial proteins along with microbial traces (Pisa et al., 2018), supporting the hypothesis of CAm as a waste repository for brain cells. In the OT, we noticed that CAm, which are characterized by Ubiquitin staining, are filled with Jagged1 puncta (Fig. 6B), indicating that they likely originate from mitral cells axons spanning the OT, where Jagged 1 is highly expressed. To further confirm their neuronal identity, we performed MAP2 and Jagged 1 colabeling and observed that all Jagged1-positive CAm were highly labeled with MAP2 (Fig. 6C). A secondary antibody control immunostaining is presented to confirm the specificity of the Jagged1 and MAP2 immunolabeling (Supplementary Fig. 5B). A chromogen immunostaining for Jagged 1 confirms that the ligand is present in the parallel fibers forming the OT and that the extracellular vesicles are immunoreactive for both Jagged1 and GAD67, which is expressed in GABAergic neurons populating the OT. This raises the possibility that CAm result from the coalescence of synaptic vesicles from mitral cells and interneurons (Fig. 6D). The selectivity of the neuronal proteins contained in the vesicles is corroborated by observing the lack of OMP labeling in the CAm (Supplementary Fig. 4C). To further validate the neuronal identity of the CAm, we performed triple immunohistochemistry for MAP2, the presynaptic marker Synaptophysin, and GFAP. We observe that CAm are all MAP2- and synaptophysin-positive, but only some CAm are ensheathed by GFAP-positive fibers (Fig. 7A insert). To further address whether CAm are presynaptic or postsynaptic, we performed double immunolabeling for Synaptophysin and PSD95. We show that most puncta are synaptophysinpositive, whereas PSD95 pixels are more sparse, and as expected, there is no pixel colocalization between PSD95 and synaptophysin (Fig. 7B; Mander's correlation coefficient $=0.02 \pm 0.01$ ). Colocalization of Jagged 1 and Synaptophysin in CAm confirms the presynaptic identity of the Jagged1 ligand (Ineichen et al., 2017; Marathe et al., 2017) (Fig. 7C; Mander's Correlation Coefficient $=0.71 \pm 0.04$ ). Taken together, CAm in the OT are derived from ending of neuronal processes both dendritic (MAP2) and axonal (Synaptophysin) and at times surrounded by GFAP-positive glial endfeet.

\subsection{Changes in CAm's density and composition with the progression of dementia}

In the medial OT of selected patient (Table 2 and Supplementary Table 1), unbiased counting of 500 CAm for over 10 randomly 
A

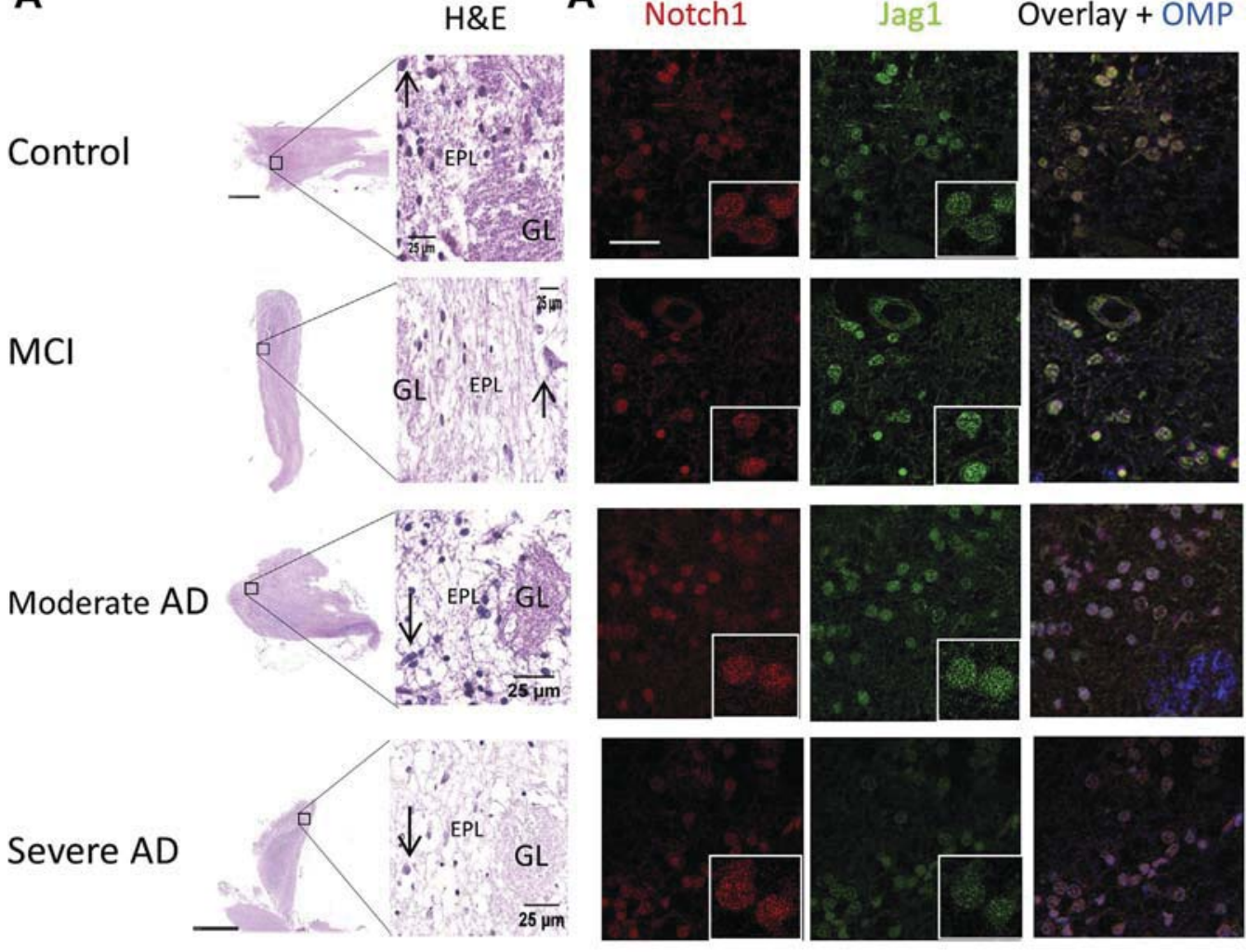

B

C
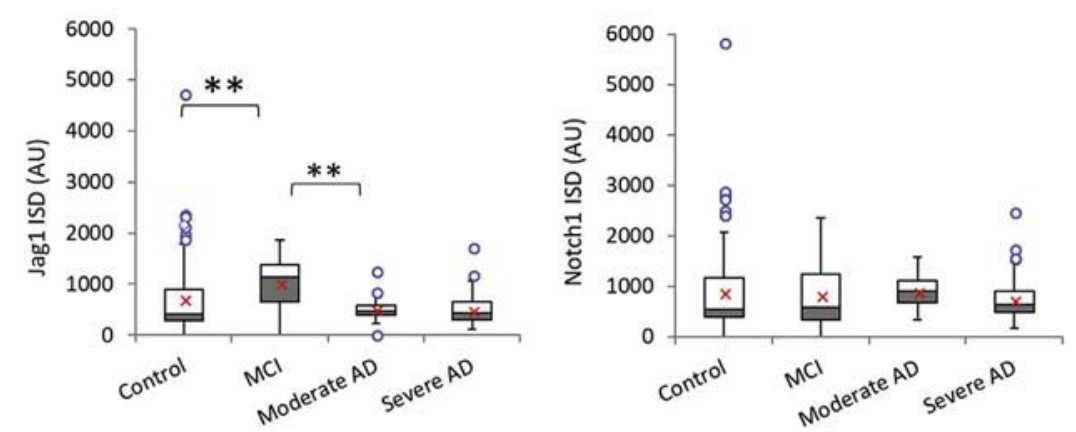

D
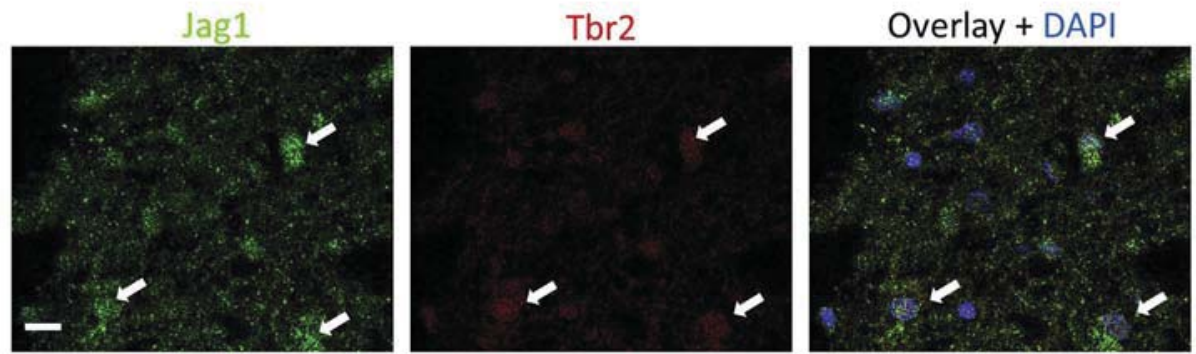

Fig. 5. Changes in signaling proteins in mitral cells with the progression of dementia. (A) Representative H\&E staining of the olfactory nerve per staging with high magnifications showing the position of the mitral cells (black arrows) in reference to the GL and the intercalating EPL. ( $A^{\prime}$ ) Triple fluorescent immunolabelings for OMP (blue) and the Notch signaling components, Notch1 (red) and Jagged1 (green), show a change in Jagged1 and OMP but not Notch1 intensities in neurons of the mitral cell layers across stages (inserts). (B) Box plot summarizing the fluorescent intensity for Jagged1 and (C) Notch1 in mitral cells. (D) Double immunolabeling confirming the expression of Jagged1 in Tbr2-positive mitral cells (white arrows) in the human olfactory bulb. On the box plot, blue empty dots are maximum and minimum outliers, and red crosses represent the mean value of the sampled data. ${ }^{* *}=p<0.01$. Scale bars in $\mathrm{A}^{\prime}$ is $25 \mu \mathrm{m}$ and in D is $10 \mu \mathrm{m}$. Abbreviations: AU, arbitrary units; EPL, external plexiform layer; GL, glomeruli; H\&E, Hematoxylin-Eosin; OMP, olfactory marker protein. (For interpretation of the references to color in this figure legend, the reader is referred to the Web version of this article.) 
A
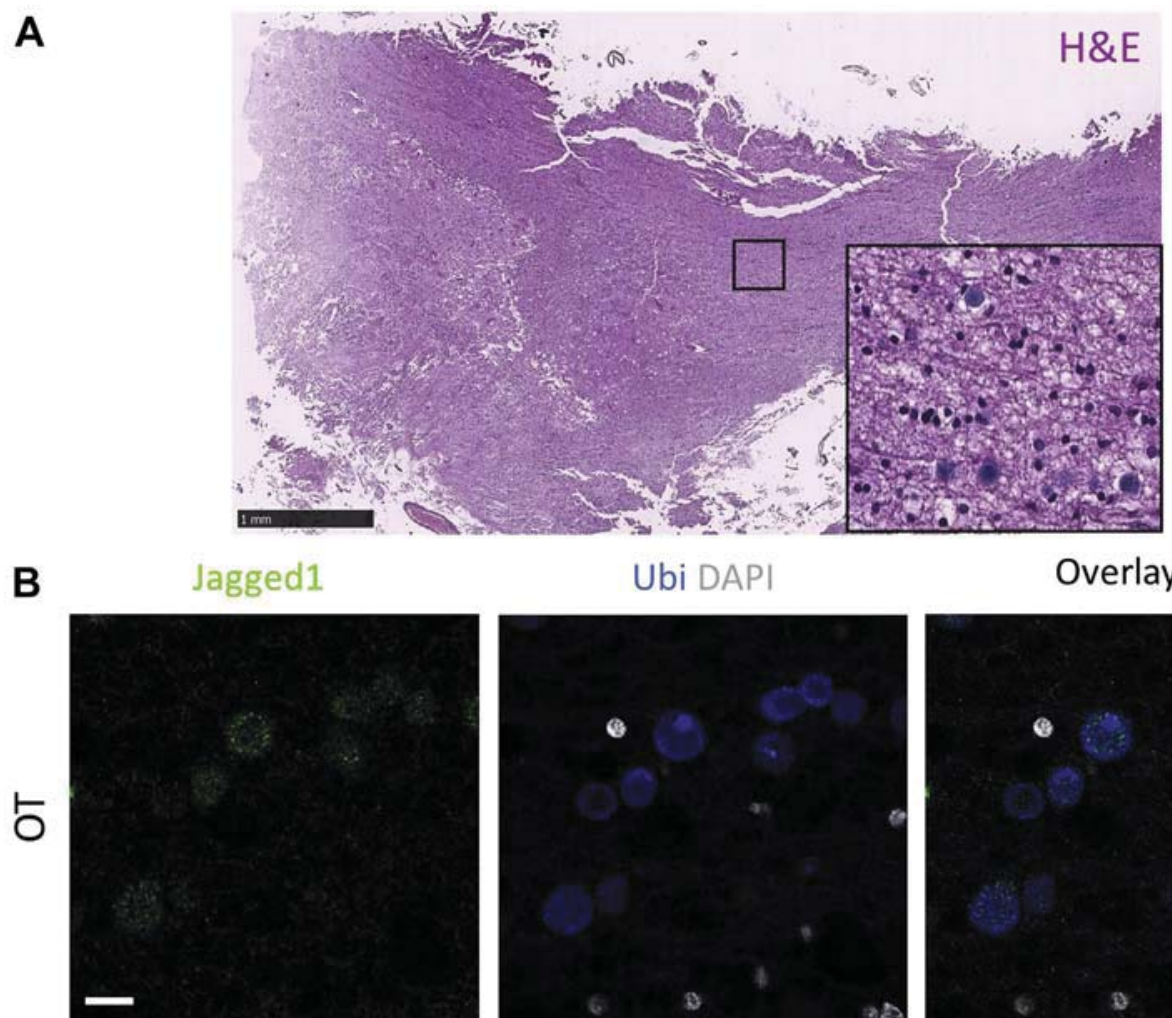

Ubi DAPI

Overlay
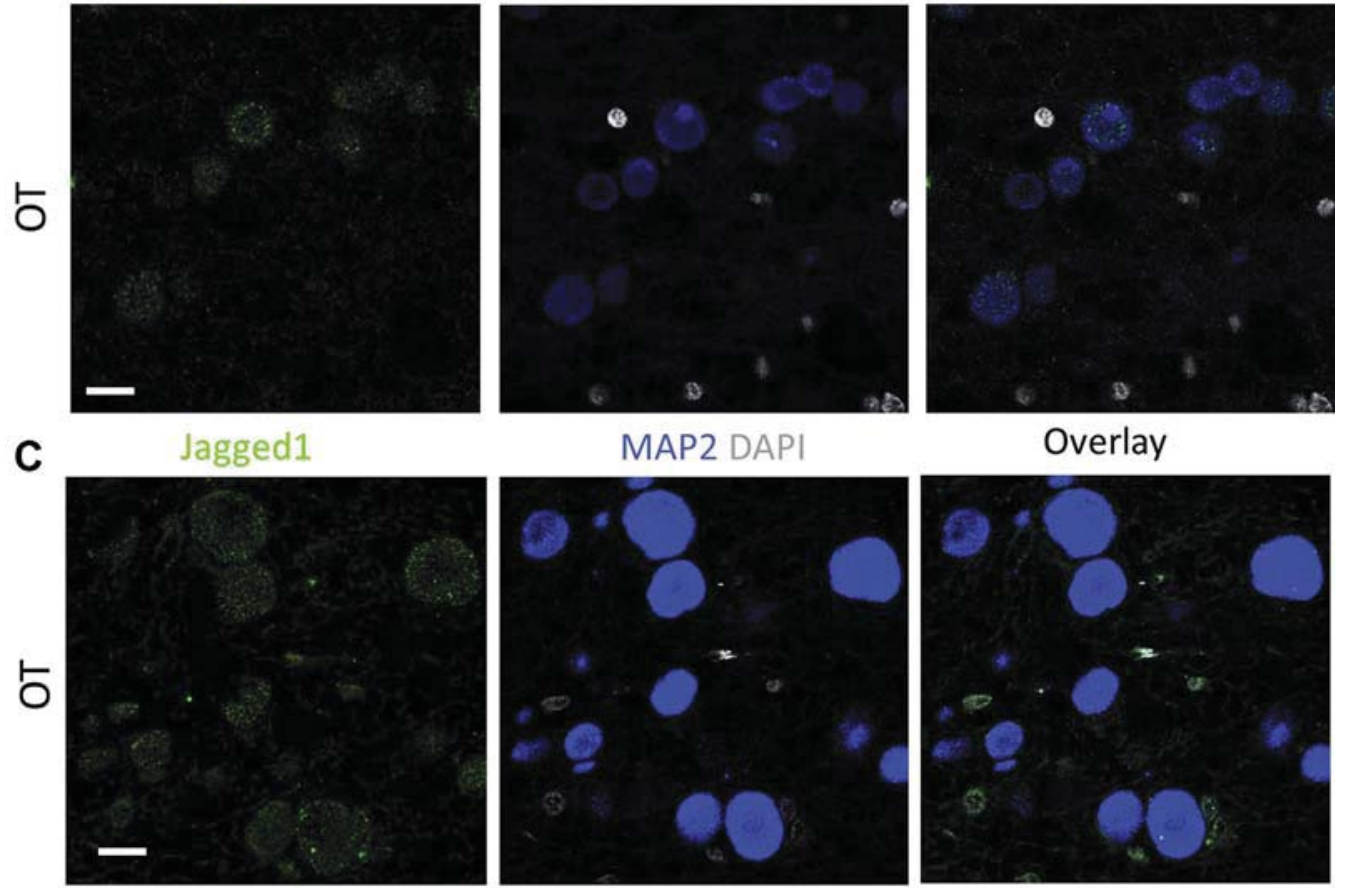

MAP2 DAPI

Overlay
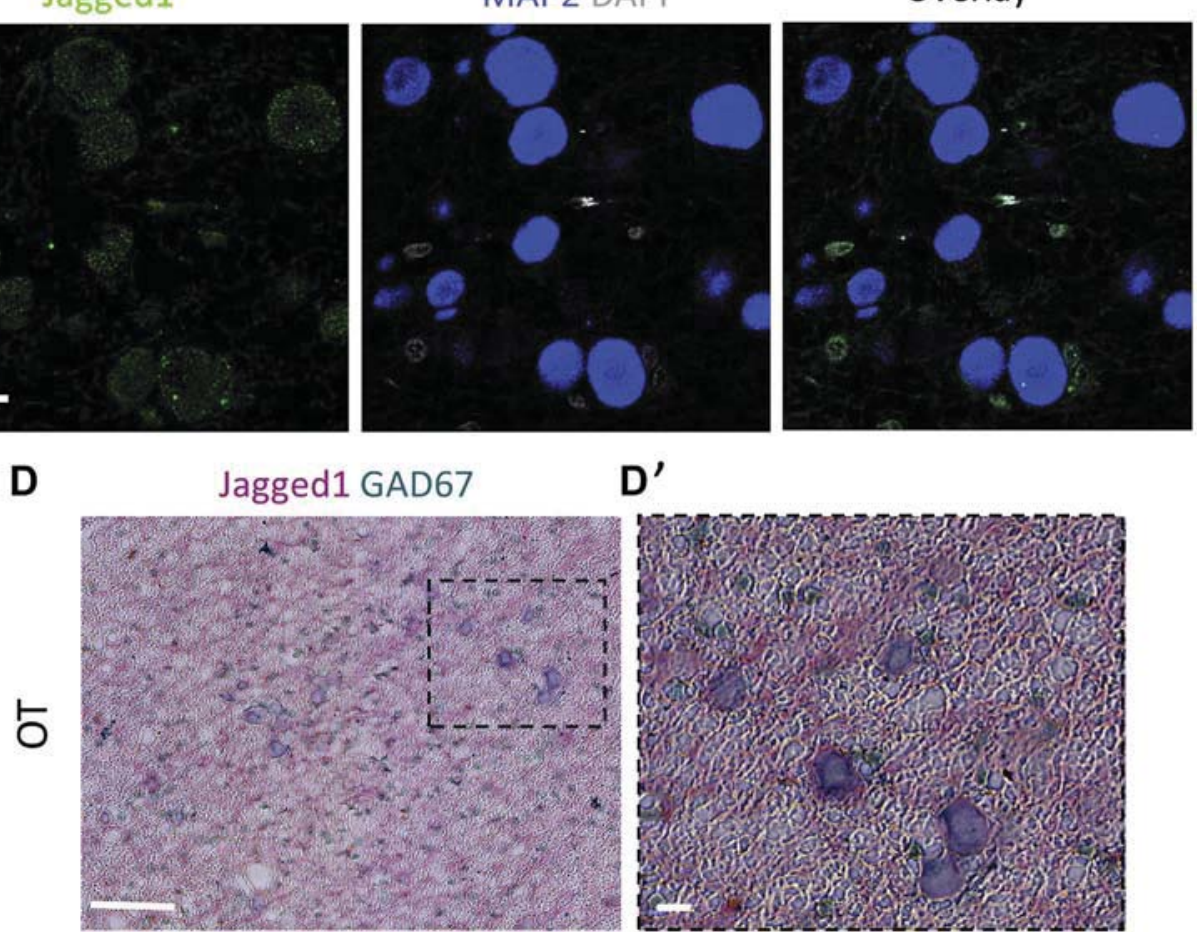

D'

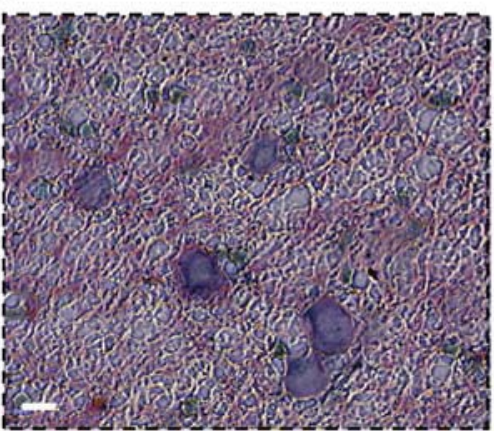

Fig. 6. Jagged1 puncta fill the CAm of the OT. (A) An abundance of CAm is found in the OT as indicated by the H\&E staining labeling the extracellular vesicles in violet (magnified insert). (B) Double immunolabeling shows Jagged1 puncta (green) restricted to ubiquitin-positive (blue) CAm, which are not DAPI (gray) positive. (C) Double immunolabeling for Jagged1 (green) and MAP2 (blue) shows the intense immunoreactivity of MAP2 and punctuate staining of Jagged1 in CAm. (D) Jagged1 (Fast red) and GAD67 (Emerald green) chromogen immunolabeling show the copresence of Jagged1 and GAD67 in CAm but not in the axonal tract or interneurons, where Jagged1 and GAD67 are separately expressed. Scale bars in A are $1 \mathrm{~mm}$, in B, C, and $\mathrm{D}^{\prime}$ are $10 \mu \mathrm{m}$, and in D are $50 \mu \mathrm{m}$. Abbreviations: CAm, corpora amylacea; H\&E, Hematoxylin-Eosin; OT, olfactory tract; GAD67, glutamate decarboxylase 67. (For interpretation of the references to color in this figure legend, the reader is referred to the Web version of this article.)

selected quadrants was conducted (Supplementary Fig. 6A-C). From MCI, we detect a significant drop in the number of CAm $\left(t_{123}=\right.$ $5.83, p<0.001$ ), which stabilizes over the course of the disease
(Table 2). In addition, we measured the diameter of the CAm to assess whether a change in size could reflect a mutation in content as previously suggested by optical molecular imaging (Galli et al., 


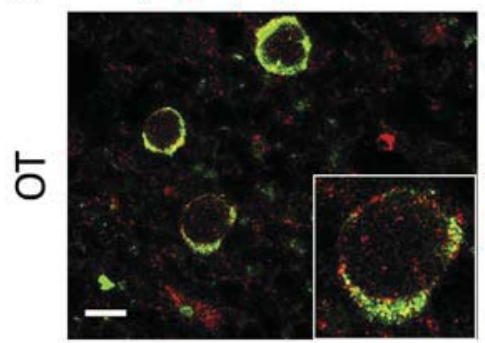

B

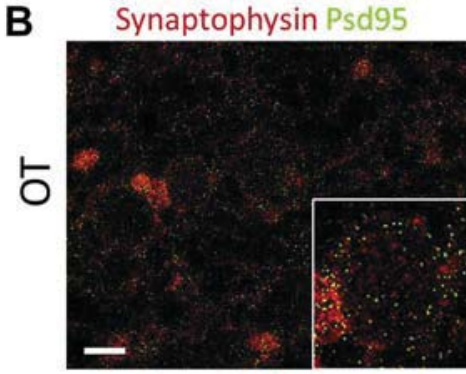

C

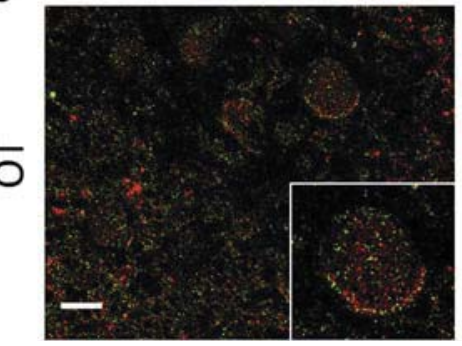

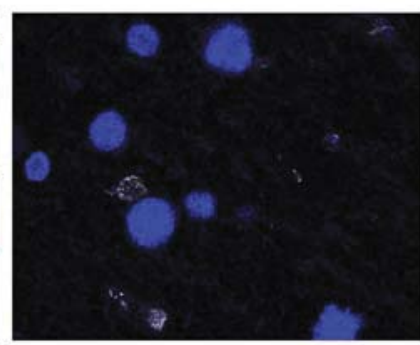

MAP2 DAPI

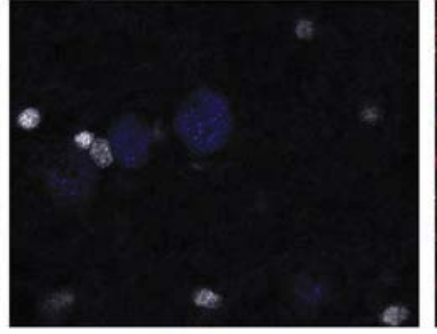

MAP2 DAPI

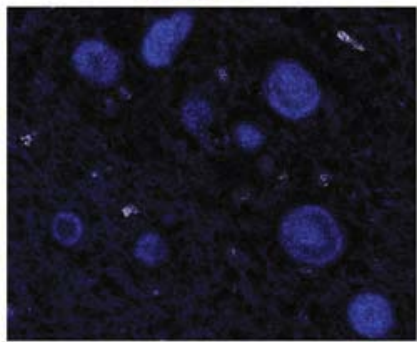

Overlay

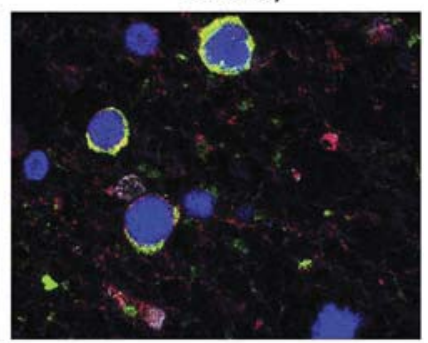

Overlay

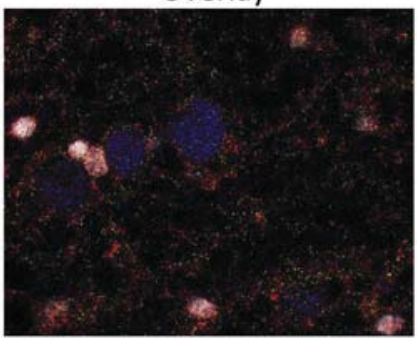

Overlay

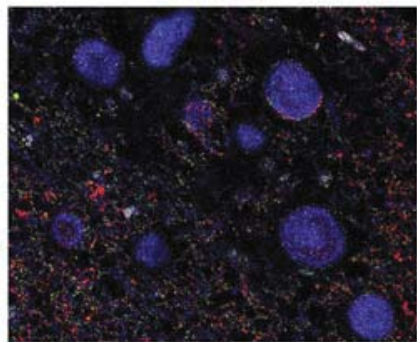

Fig. 7. Synaptic identity and astroglia reactivity of the CAm in the human olfactory nerve. (A) Triple fluorescent immunolabeling for Synaptophysin (red), GFAP (green), and MAP2 (blue) shows specific staining of the presynaptic marker, Synaptophysin, while GFAP-positive fibers encircle some extracellular components, which are all MAP2-positive. (B) Fluorescent immunolabeling for Synaptophysin (red), PSD95 (green), and MAP2 (blue) indicates that sparsity of PSD95 puncta inside the Synaptophysin and MAP2-positive CAm. (C) Representative image displaying triple immunolabeled CAm with Jagged1 (green), synaptophysin (red), and MAP2 (blue). Scale bar in all panels is $10 \mu \mathrm{m}$. Abbreviations: CAm, corpora amylacea; GFAP, glial fibrillary acid protein. (For interpretation of the references to color in this figure legend, the reader is referred to the Web version of this article.)

2018). From our unbiased analysis (Supplementary Fig. 6C), the size of the CAm remains unchanged between the diseased and healthy control specimen (Table 2). In line with other works (Notter and Knuesel, 2013), Reelin-positive CAm are either hollow or filled, with a prevalent accumulation of the protein at the border (Fig. 8A) and the specificity of the immunolabeling is confirmed by secondary antibody control (Supplementary Fig. 5B). This suggests that Reelin occurs in 2 forms: soluble and membrane-bound. On the other hand, Jagged1 puncta fill the CAm's inner space, indicating a uniquely soluble form for Jagged 1 in these structures (Fig. 8A). Colocalization analysis indicates that Jagged 1 and Reelin have only partially overlapping patterns (Fig. 8A and Supplementary Table 2). Notch1 labels the processes on the OT and the membrane of the CAm, partially coexisting with Reelin (Fig. 8A and Supplementary Table 2) but, as expected, in a complementary pattern to Jagged 1 (Supplementary Table 2). To understand whether the content of the signaling ligands changes through the progression of dementia, we performed an integrated signal density analysis for Jagged1 (Supplementary Fig. 6D-F) and Reelin. We observed that CAm express a spectrum of intensities of Jagged 1 and Reelin, likely reflecting the load of the signaling modulators (Fig. 8B-D). The distribution of intensities for Jagged 1 narrows down in $\mathrm{MCI}(\mathrm{KS}, \mathrm{D}=$ 0.2996 and $p=0.000)$, remains unchanged in moderate $\mathrm{AD}(\mathrm{KS}, \mathrm{D}=$ 0.0881 and $p=0.327$ ), and declines further in severe AD (KS, D = 0.1938 and $p=0.000$ ) (Fig. $8 \mathrm{E}$ ). At the same time, Reelin expression is visibly lower in moderate and severe AD (Fig. 8D). Fluorescent intensity analysis indicates also for Reelin a progressive reduction of signal intensities from the moderate $\mathrm{AD}(\mathrm{KS}, \mathrm{D}=0.2598$ and $p=$ $0.000)$ and a further decline in severe $\mathrm{AD}(\mathrm{KS}, \mathrm{D}=0.3775$ and $p=$ 0.000 ), whereas $\mathrm{MCI}$ and controls appear comparable (KS, D = 0.0441 and $p=0.987$ ) (Fig. 8F). Along with the changes in Jagged1 and Reelin, we detect an ectopic expression of MAP2 in CAm (Supplementary Fig. 7A) and an apparent reduction in tau and GFAP labeling (Fig. 9A). Quantitative analysis confirms a deviation in MAP2 expression pattern in CAm from MCI and moderate stage (KS, $\mathrm{D}=0.3596$ and $p<0.0001$ ) followed by a reversal reduction in severe patients (KS, D $=0.3483$ and $p=0.000$ ) (Supplementary Fig. 7B). Inversely to MAP2, tau expression, which is typically found surrounding the CAm, decreases from the MCI stage (KS, D = 0.3366 and $p<0.0001$ ) and decays further in severe $\mathrm{AD}(\mathrm{KS}, \mathrm{D}=$ $0.5644 p<0.0001$ ), whereas MCI and moderate AD appear indistinguishable (Fig. 9B). Finally, the GFAP enwrapping is diminished in $\mathrm{MCI}(\mathrm{KS}, \mathrm{D}=0.3014$ and $p=0.000)$ and is further decreased in the severe $\mathrm{AD}$ (KS, $\mathrm{D}=0.4795$ and $p=0.000$ ) (Fig. 9C), suggesting a change in the dynamics of CAm formation and their immunoreactivity. Considering the abundance of NFTs and p-tau positive neuropils along the axonal bundles of the OT (Fig. 2), and the apparent neuronal identity of the CAm, we asked whether the reduction in tau protein in the CAm was a result of microtubule breakdown due to tau hyperphosphorylation. We show that with the increasing severity of dementia, CAm becomes more tangled as indicated by the increase in p-tau immunoreactivity (Fig. 9D and E). These pieces 
Table 2

Morphological analysis of CAm in OT

\begin{tabular}{lllll}
\hline & CTL $(\mathrm{n}=7)$ & $\mathrm{MCI}(\mathrm{n}=6)$ & $\begin{array}{l}\text { Moderate } \\
\mathrm{AD}(\mathrm{n}=7)\end{array}$ & $\begin{array}{l}\text { Severe } \\
\mathrm{AD}(\mathrm{n}=6)\end{array}$ \\
\hline Density $\left(\mathrm{CA} / 100 \mu \mathrm{m}^{2}\right)$ & $36.9 \pm 8.2^{\mathrm{a}}$ & $19.9+5.5$ & $27.8+4.7$ & $20.9+5.6$ \\
Diameter $(\mu \mathrm{m})$ & $14.5 \pm 0.3$ & $16.3+0.9$ & $13.6+0.3$ & $14.1+0.1$
\end{tabular}

Key: AD, Alzheimer's disease; CAm, corpora amylacea; CTL, control; MCI, mild cognitive impairment; OT, olfactory tract. a $p<0.01$.

of evidence indicate dynamic changes in the molecular composition of CAm in the progression of dementia.

\section{Discussion}

\subsection{Progressive tau and amyloid pathology in the olfactory nerve of patients with $A D$}

In ND, hyposmia can be considered a prodromal symptom of cognitive decline and patients with no dementia, scoring low for the olfactory identification test, bare a higher risk of developing cognitive symptoms in a 2-year follow-up (Devanand et al., 2014). Reduction in the $\mathrm{OB}$ volume is detected already in patients with MCI (ter Laak et al., 1994; Thomann et al., 2009). However, bulbar atrophy as a result of early neuronal loss remains disputed (Mundiñano et al., 2011; Servello et al., 2015). Furthermore, it remains unclear how olfactory transmission deficit may anticipate neuronal network dysfunction in higher brain centers such as the hippocampus and entorhinal cortex that undergo neurodegeneration (Brai and Alberi, 2018). In our study, we see a distinct pathology between the regions of the olfactory system (OB, OT, and $A O N$ ) in $A \beta$ depositions, fibrillary neuropils, and NFTs. Although the $A \beta$ immunoreactivity is seen as classical ring with core-like deposits in the cortical tissue, in all olfactory nerve regions considered, the depositions are more diffused suggesting intracellular $A \beta$ 42 accumulation specifically in the distal neurites compartment (Brai and Alberi, 2018; LaFerla et al., 2007). In the healthy subjects, such depositions can be rarely seen in the GL, but they are clearly evident with progression of dementia. Such a diffuse pattern is also visible in the AON, but it is more prominent in the GL region.

On the contrary to the $A \beta$ depositions, tau pathology is more severe with depositions of neuropil threads and NFTs in all of the 3 areas examined. The severity of these aggregates increase with the progression of disease, and NFT formation can be seen much earlier when compared with the amyloid pathology in this region. In agreement with the previous studies (Attems et al., 2014; Kovács, 2004; Risacher et al., 2017), our data confirm the prominent and early tauopathy in the olfactory nerve.

\subsection{Reactive astrogliosis in the $\mathrm{OB}$ and tract in the early to moderate phases of $A D$}

With aging and $\mathrm{AD}$, on exposure to $\mathrm{A} \beta$ oligomers and to free fatty acid from neuronal membrane breakdown, astroglia increase their proinflammatory profile, producing reactive oxygen species and propagating a neuroinflammatory response.

Alteration to the neuroglia GL network can be tracked in our study by the slightly increased GFAP immunoreactivity at the MCI stage, coinciding with the appearance of the first p-tau-positive neuropil, likely representing disaggregating sensory axon terminals. While the p-tau and amyloid pathology rise in the GL, from the moderate stage onward, astrogliosis dissipates, suggesting a progressive atrophy of the neuroglia connections, which may affect olfactory transmission (Roux et al., 2011). Loss of astrocytes has been previously reported in the medial prefrontal cortex of a triple transgenic AD mouse model demonstrating no correlation between astrogliosis and plaque accumulation in some regions of the brain (Kulijewicz-Nawrot et al., 2012; LaFerla et al., 2007). Furthermore, the diffuse morphology of the $A \beta$ plaques in the GL at the moderate and severe stage, suggest that $A \beta$ may be intracellular/intraxonal, causing neuronal demise without triggering an astroglia reaction (Gouras et al., 2005; LaFerla et al., 2007). In support of this notion, a former study confirms the low astrogliosis response around diffused plaques (Mathur et al., 2015). While resident astroglia of the OT are thought to be silent, reactive astrogliosis in the OT has been reported in patients affected by AD, PD, and FTD (Kohl et al., 2017). Our study confirms a prominent astrogliosis in the OT of $\mathrm{AD}$ patients with a peak of reactive glia in the moderate stage, which resolves afterward. Microgliosis recapitulates astrogliosis in its dynamic expression and prevalence in the OT region (data not shown). The timing of astrogliosis correlates with the accumulation of NFTs in the OT but not with the maximal tau pathology possibly indicating an astroglia atrophy subsequent to its reactive phase. Taken the role of glia in synaptic modulation also in the bulb (Roux et al., 2011), we can hypothesize that the transient increase in reactive astrogliosis may contribute to the olfactory transmission deficits developing with dementia.

\subsection{Altered Jagged 1 expression in human olfactory system with dementia}

The accumulation of p-tau in the olfactory system reflects a loss in axonal integrity and stability impairing the transdiffusion of many synaptic proteins and modulators (Liu et al., 2012). In our previous article, we reported the displacement of Notch1 from neurons to fibrillary plaques and NFTs in the parenchyma of sporadic AD brains (Brai et al., 2016). Similarly, in the OT, we observe a colocalization of Notch1 in fibrillary fibers, indicating a general enrichment of the signaling receptor in neuritic varicosities. Nevertheless, when analyzing the expression of Notch1 in mitral cells, we did not observe a reduction in Notch1 in this neuronal population among the different stages of dementia. This was surprising, taken the role of Notch 1 in olfactory plasticity in rodents (Brai et al., 2014) and its reduction in pyramidal hippocampal and cortical neurons in AD specimen (Brai et al., 2016). This emphasizes the diversity between species and the different biological ontogeny of excitatory networks between sensory and central areas. On the other hand, Jagged 1 expression in mitral cells changes across the development of dementia. In $\mathrm{MCI}$, we observe an increase in Jagged1 possibly indicating an increase in synaptic drive that is known to augment Jagged1 levels (Alberi et al., 2011). In support of this hypothesis, a recent article reported an increase in dopaminergic glomerular interneurons in AD brains. This cell population modulates the synchronous firing of mitral cells, influencing transmission and activity-dependent processes, such as Jagged1 expression. Increased Jagged1 expression in the olfactory mitral cells of the patients with AD can also be due to the increase in BACE1 activity (Alberi et al., 2011; Cheng et al., 2014; Yoo et al., 2017), a secretase responsible for regulating Jagged1 turnover (Hemming et al., 2009). With the increasing severity of dementia, Jagged 1 expression in mitral cells declines indicating that the Notch ligand may reflect ongoing synaptic dysfunction in the olfactory nerve. This is supported by our and previous reports implicating Jagged 1 in neuronal plasticity (Alberi et al., 2011; Wang et al., 2004), and olfaction (Brai and Alberi, 2015) and a progressive decline in Jagged 1 in the brains and cerebrospinal fluid of patients with AD (Marathe et al., 2017). Thus, Jagged 1 in mitral cells appears to follow the pathogenesis of neurodegeneration closely reflecting surrounding network changes. 
A

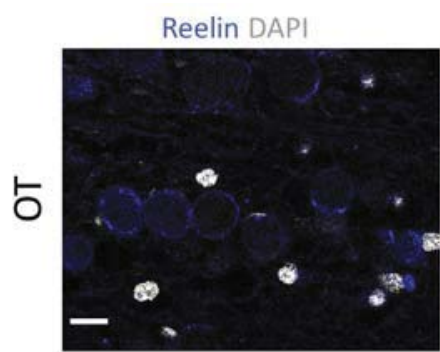

Notch1 Jag1

Overlay

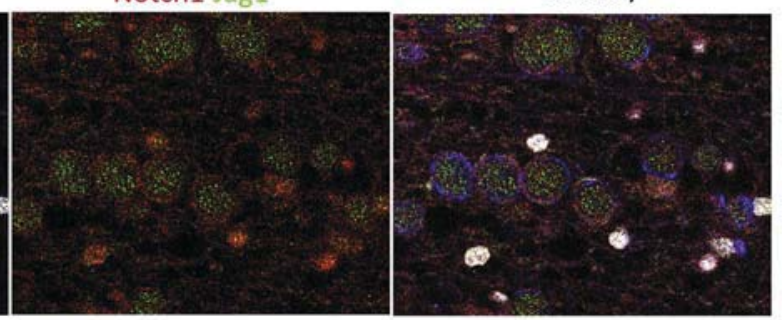

B Jagged1 DAPI

C Jagged1
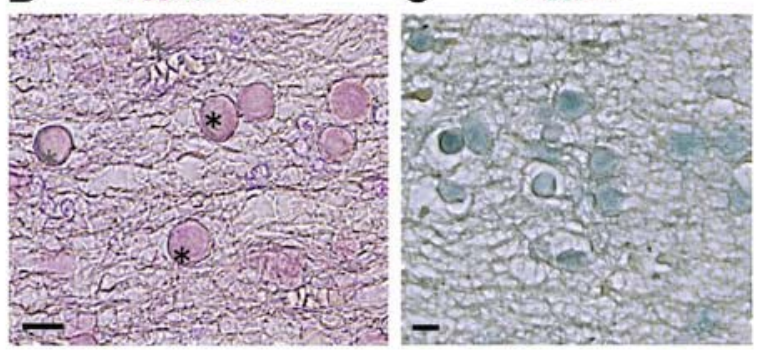

D

CTL

$\mathrm{MCl}$
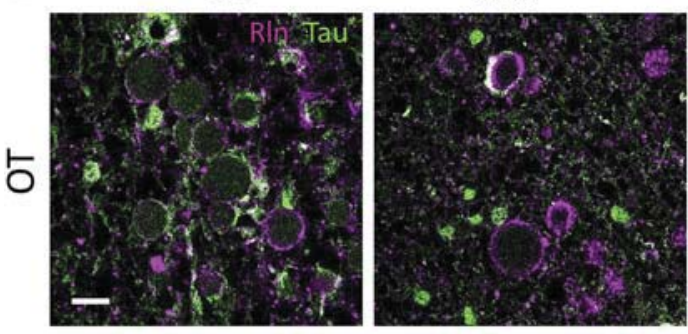

Moderate AD
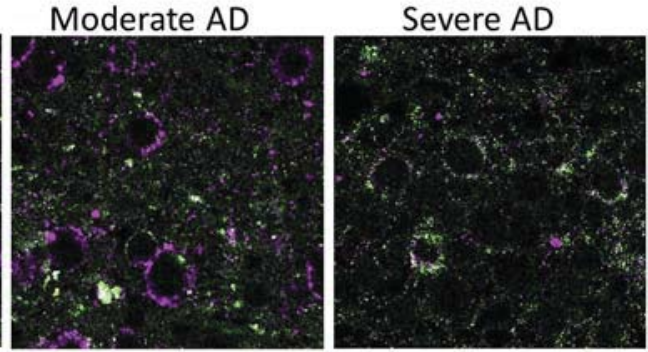

E

F
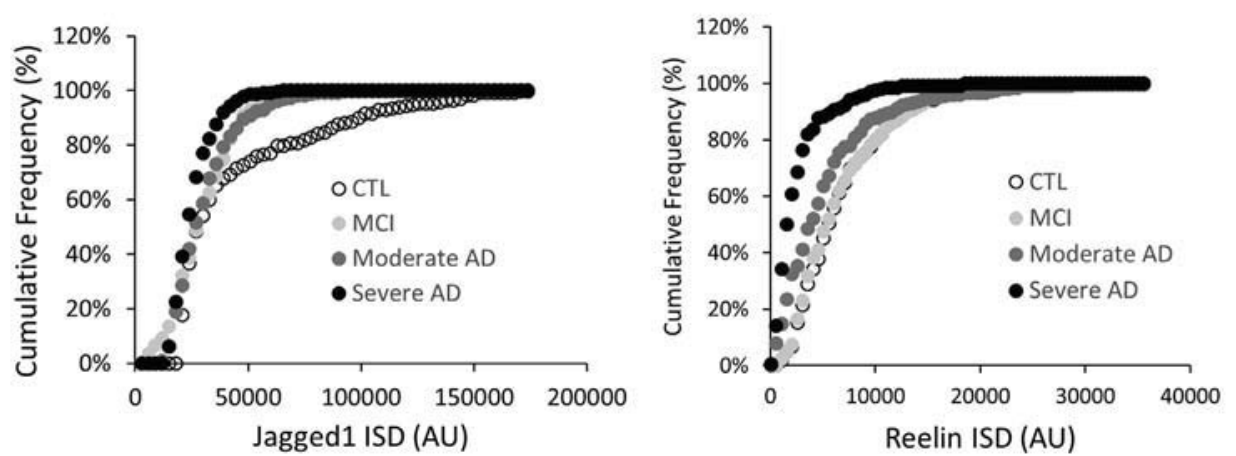

Fig. 8. Signaling modulator Jagged1 and Reelin are enriched in CAm and their expression changes with the progression of dementia. (A) Fluorescence immunohistochemistry indicates that CAm express Reelin (blue) and are filled with Jagged1 puncta (green), whereas Notch1 (red) is localized to the border region and overlaps with Reelin. (B) Fast-Red chromogen immunohistochemistry for Jagged1 overlaid with DAPI (blue) shows the CAm filled with pink chromogen at different degrees of intensity (gray star indicates low levels of Jagged1 and black star high level of Jagged1). (C) Emerald green chromogen immunolabeling shows the different intensities of Jagged1 in CAm using a monoclonal antibody. (D) Representative fluorescent immunohistochemistry for Reelin (magenta) and tau (green) at each staging of dementia displays a progressive change in expression with the progression of the disease. Cumulative probability plot shows the differential distributions of (E) Jagged1 and (F) Reelin signal intensities in $\mathrm{MCI}$, moderate $\mathrm{AD}$, and severe $\mathrm{AD}$ as compared to healthy controls. Scale bars in all panels are $10 \mu \mathrm{m}$. Abbreviations: AU, arbitrary units; CAm, corpora amylacea; ISD, integrated signal density. (For interpretation of the references to color in this figure legend, the reader is referred to the Web version of this article.)

4.4. Changes in cellular signaling ligands and cytoskeletal proteins with the progression of dementia

Along with the expression of Jagged 1 in mitral cells body, we found that it localized to the axonal tracts in the OT as well as in CAm populating this region. In the CAm, Jagged1 appears in puncta filling their interior, suggesting a soluble form of the protein as a result of shedding from the membrane surface (Nehring et al., 2005). Soluble Jagged1 has been reported to induce synaptic potentiation in animal models and may be considered a signaling neuromodulator (Alberi et al., 2011; Wang et al., 2004). Mitral Jagged 1 may be released within the extracellular vesicles forming the CAm. Further supporting this hypothesis, Jagged1 colocalized with the presynaptic marker, synaptophysin, strengthening the synaptic origin of CAm. Along with Jagged 1 expression in the CAm, we also find GAD67, which marks GABAergic neurons populating the OT region (Saiz-Sanchez et al., 2016). Interneurons increase synchronous firing of mitral cells (Schoppa, 2006) and are 
A
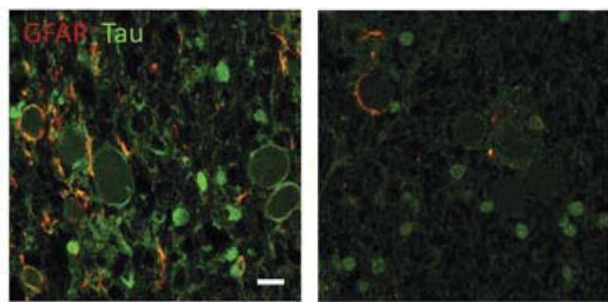

B

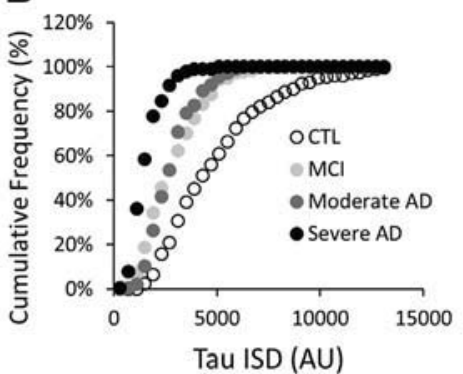

D

CTL

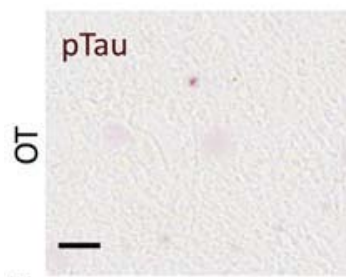

E

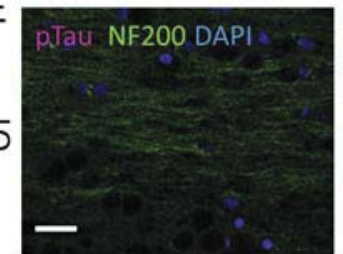

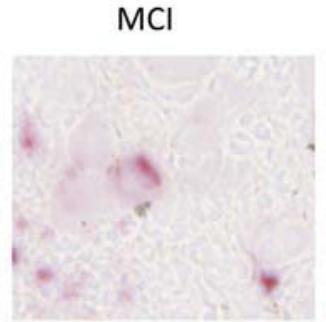

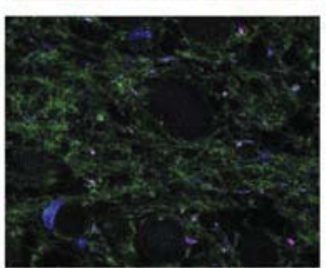

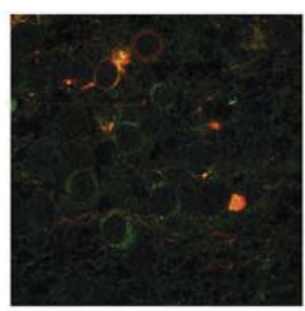

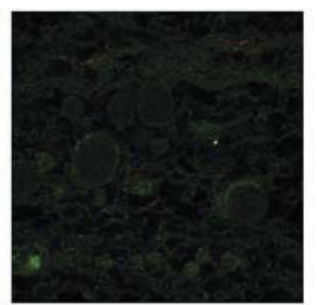

C

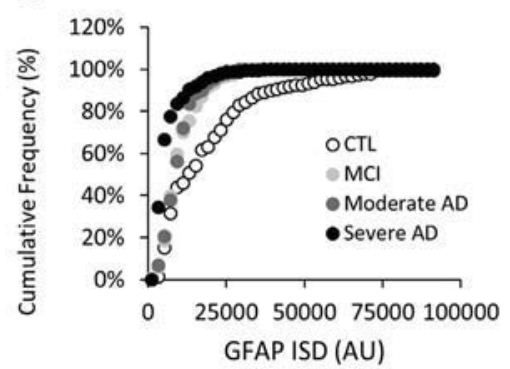

Moderate AD

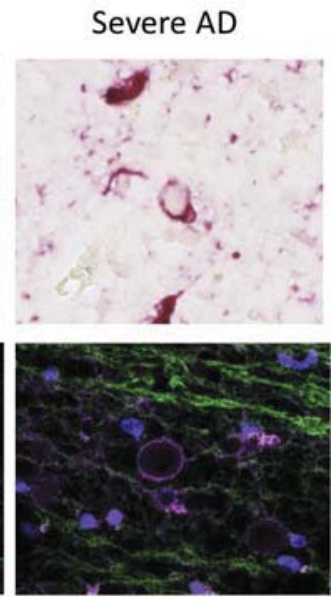

Fig. 9. Shift in cytoskeletal content of CAm in dementia. (A) Double immunofluorescence staining for GFAP (red) and tau (green) shows a decrease in the expression of GFAP and tau in CAm with the progression of dementia. Cumulative probability plots for (B) tau and (C) GFAP summarize the distribution of intensities along with the increasing severity of the disease. (D) p-tau chromogen immunohistochemistry indicates that with the increased severity of the disease, CAm are surrounded by p-tau positive tangles. (E) Fluorescent immunohistochemistry for p-tau and NF200 confirms the increase in p-tau accumulation around the CAm, whereas their inner remains devoid of any aggregate. Scale bars in A are $20 \mu \mathrm{m}$ and D-E and G are $10 \mu \mathrm{m}$. Abbreviations: CAm, corpora amylacea; p-tau, phosphorylated tau; OT, olfactory tract. (For interpretation of the references to color in this figure legend, the reader is referred to the Web version of this article.)

essential in homeostatic scaling and olfactory discrimination (Giridhar et al., 2011). In AD, a decline in Somatostatin interneurons has been reported in the piriform cortex (Li et al., 2010) and AON (Saiz-Sanchez et al., 2010) but less is known about the viability of the interneurons in the olfactory nerve (SaizSanchez et al., 2016). The presence of GAD67 in CAm suggests that these structures result from the coalescence of extrasynaptic vesicles likely originating from both inhibitory and excitatory neurons, which is supported by the presence of parasynaptic CAm in the thalamus of patients with $\mathrm{AD}$ and Huntington's disease (Averback, 1981). The neuronal origin of CAm is further corroborated by the presence of the cytoskeletal axonal and dendritic markers, tau and MAP2, labeling, respectively, its perimeter and its core, and the ensheathing layer of GFAP from astroglia typically sealing the synaptic terminals (Osborn et al., 2016). Our finding is in line with a previous article showing that CAm are enwrapped by GFAP-positive fibers (Pirici et al., 2014). Interestingly, Pirici et al indicate that CAm are part of larger 3-dimensional meshed network with tunnels connecting the extracellular bodies and question whether these extracellular structures are constitutive resulting from the accumulation of cellular and extracellular components without a specific neuronal or glia origin. Another more recent article argues against the presence of many of the previous identified proteins in CAm, such as GFAP, S100, AQP4, NeuN, or class III $\beta$-tubulin, showing that the enrichment in IgM in CAm may cause nonspecific labeling by contaminating IgM traces present in commercially available primary and secondary antibodies (Augé et al., 2017). We cannot confirm this statement, as the patterns of the proteins analyzed diverge substantially from each other, that is, the GFAP-positive sheet localize at the rim of CAm, punctuated synaptic proteins decorate their center, and MAP2 fills the CAm. Furthermore, all our chromogen and fluorescent immunolabeling were conducted using a secondary antibody control, which revealed no immunoreactivity on CAm or other structures That said, the IgM immunoreactivity shown by Auge and colleagues reveals an ubiquitous and uniform arrangement characteristic of the neoepitopes (Augé et al., 2017), which should be taken into account when observing a similar pattern. The presence of neuronal proteins is further corroborated by the latest proteomic analysis of the CAm fractions, revealing a 
complex proteome in CAm including both cytoskeletal, synaptic, and neuronal and glial components (Pisa et al., 2018). Surprisingly, neither Reelin nor Jagged 1 was found in these CAm extracts. This can be explained either by the low levels of the neurosignaling modulators in the insular cortex from which CAm were obtained or the known detection limits of the liquid chromatography- mass spectrometry analysis. Nevertheless, in the CAm of the OT, Reelin, which is expressed both in mitral cells (Okuyama-Yamamoto et al., 2005) and interneurons (Herrmann et al., 2008), decorates the perimeter and the center of the CAm. Recent studies have shown that Reelin-positive CAm in the hippocampus increase with the severity of AD (Notter and Knuesel, 2013), and increased Reelin expression has been reported in the cerebrospinal fluid from patients with AD and FTD (Sáez-Valero et al., 2003). On the other hand, another article shows that Reelin levels decline with the progression of dementia in neurons of the frontal cortices, hippocampus, and entorhinal cortex (Herring et al., 2012). In both cases, Reelin turnover is affected by the onset of ND and behaves similarly to Jagged1, as we previously reported (Marathe et al., 2017). In our present findings, we report that both Jagged 1 and Reelin intensities decline in CAm with the severity of dementia. Taken the established role of Reelin and Jagged 1 as neuroplasticity modulators (Brai et al., 2015; Trotter et al., 2013; Weeber et al., 2002), our findings infer impaired proteolytic processing and synaptic function. Few studies have also shown myelin-positive small neuronal extrusions ranging from 1 to $3 \mu \mathrm{m}$ in size from the axons and dendrites in response to immune challenge that contain Reelin, fibrillary proteins, sAPP, and A $\beta$ (Doehner et al., 2010), which was confirmed in human brain specimen (Notter and Knuesel, 2013). On the other hand, in our study, we could not detect any $\mathrm{A} \beta$ peptides in the CAm of the OT, but we show that the strong tau expression, defining the vacuolar geometry of CAm, dissipates with the progression of dementia. Tau expression was previously reported in CAm of the cortex, hippocampus, and optic nerve (Day et al., 2015; Loeffler et al., 1993; Notter and Knuesel, 2013; Tate-Ostroff et al., 1989) and was further confirmed by the proteomic analysis of CAm (Pisa et al., 2018). The observed decline in tau expression in CAm of the OT may reflect neuritic varicosity disaggregation supported also by their decline in density and the increase in p-tau expression at the CAm's border. Previously, p-tau expression could not be detected in CAm of the hippocampus (Notter and Knuesel, 2013), suggesting probably differential biodynamics among the brain regions or divergence in experimental protocols. Importantly, GFAP labeling around CAm marks reactive astrocytic endfeet (Doehner et al., 2012) likely confining abnormal accumulation of toxic proteins within the extraneuronal varicosities (Knuesel et al., 2009; Singhrao et al., 1995). In cases of severe $\mathrm{AD}$, we hardly see any GFAP-positive CAm, inversely to the p-tau depositions decorating the CAm.

The progressive p-tau immunoreactivity of the CAm and the counterpart decline in surrounding GFAP is aligned with a loss of the protective role of ensheathing astroglia. Overall, we hypothesize that the accumulation of neuronal and non-neuronal proteins in the CAm represents an intraneuronal protein extrusion mechanism caused by impaired proteasomal or autophagosomal clearance or due to failure in transport mechanisms observable as early as in MCI. The change in the content of olfactory CAm is matched by an early reduction in their density. This finding is in contrast with other reports indicating an increase of CAm in limbic regions from patients with $A D, P D$, multiple sclerosis, temporal lobe epilepsy, and so forth (Augé et al., 2017). Nevertheless, few other studies focusing on disorders related to sensory and motor systems report a decrease in CAm in the optic nerve with the severity of glaucoma (Kubota and Naumann, 1993) and a reduction in CAm in the anterior horn of the spinal cord from patients with amyotrophic lateral sclerosis (Cavanagh, 1998). Furthermore, a decline of CAm may also reflect an axonal atrophy of the OT, supported by 2 studies showing a 30\% decrease in axonal density (Armstrong et al., 2008) and 52\% loss of myelinated axons in the OT of patients with AD (Davies et al., 1993). Altogether, taken the dynamic changes undergoing in CAm, it is likely that their density drops along with the shift in neuromodulator and cytoskeletal contents reflecting a dysfunction of the tripartite synapse in the OT.

\section{Conclusion}

The olfactory system has been extensively studied in rodents and has received much interest in the clinical setting based on the incidence of olfactory impairment in a variety of ND. Nevertheless, the understanding of the molecular and structural changes occurring in this central sensory structure in humans remains limited. This article addresses progressive changes in known hallmarks of $\mathrm{AD}$, signaling molecules, and cytoskeletal markers occurring in the olfactory nerve. We have determined that CAm in the parenchymal OT are of neuronal origin, containing synaptic markers, neurosignaling molecules, and cytoskeletal proteins with an ensheathing glial layer. In addition, we report a decline in the CAm's density and significant changes in their content starting from the MCI stage. This suggests that sensory nerves undergo an early turnover that correlates with the onset of the cognitive symptoms. Furthermore, based on the neuronal origin of CAm in this region, we show that alterations in CAm content are dynamic and may reflect ongoing synaptic changes affecting olfaction. Our study is limited by the absence of records for chemosensory tests that can be matched to the structural and molecular findings. In addition, our small number of subjects and the sparse information available on the human olfactory nerve do not allow for a thorough comparative analysis at this time. Taken the importance of the olfactory system in the diagnostics and therapeutics of ND, we foresee that this field will expand in the next future allowing for a better understanding of the early olfactory markers as a readout of sensory and central network interplay.

\section{Disclosure}

The authors declare that they have no competing interests.

\section{Acknowledgements}

The authors would like to gratefully acknowledge all donors and their families for the tissue provided for this study. Human tissue samples were supplied by the Brains for Dementia Research program, jointly funded by Alzheimer's Research UK, the Alzheimer's Society and the Medical Research Council, and sourced from the Oxford Brain Bank. The Oxford Brain Bank is also supported by the National Health Service, United Kingdom. The authors are also grateful to Mrs. Carolyn Sloan in helping them selecting the patients' specimen.

This project is supported by funds of the Swiss National Science Foundation (N.163470) and the Ministry of Science and Education Switzerland (ESKAS No. 2017.0480).

Authors' contributions: $\mathrm{PB}$ conducted the experiments and wrote part of the article; AM conducted some of the analysis; $\mathrm{MJ}$ assisted in the experimentation and performed some image captions; EB contributed to the writing; and LA designed the study and wrote the manuscript.

The data sets used and/or analyzed during the present study are available from the corresponding author on reasonable request. 


\section{Appendix A. Supplementary data}

Supplementary data associated with this article can be found, in the online version, at https://doi.org/10.1016/j.neurobiolaging.2018. 12.006 .

\section{References}

Alberi, L., Hoey, S.E., Brai, E., Scotti, A.L., Marathe, S., 2013. Notch signaling in the brain: in good and bad times. Ageing Res. Rev. 12, 801-814.

Alberi, L., Liu, S., Wang, Y., Badie, R., Smith-Hicks, C., Wu, J., Pierfelice, T.J., Abazyan, B., Mattson, M.P., Kuhl, D., Pletnikov, M., Worley, P.F., Gaiano, N., 2011. Activity-induced notch signaling in neurons requires Arc/Arg3.1 and is essential for synaptic plasticity in hippocampal networks. Neuron 69, 437-444.

Arendt, T., Bigl, V., Arendt, A., Tennstedt, A., 1983. Loss of neurons in the nucleus basalis of Meynert in Alzheimer's disease, paralysis agitans and Korsakoff's disease. Acta Neuropathol. 61, 101-108.

Armstrong, R.A., Syed, A.B., Smith, C.U.M., 2008. Density and cross-sectional areas of axons in the olfactory tract in control subjects and Alzheimer's disease: an image analysis study. Neurol. Sci. 29, 23-27.

Attems, J., Walker, L., Jellinger, K.A., 2014. Olfactory bulb involvement in neurodegenerative diseases. Acta Neuropathol. 127, 459-475.

Augé, E., Cabezón, I., Pelegrí, C., Vilaplana, J., 2017. New perspectives on corpora amylacea in the human brain. Sci. Rep. 7, 41807.

Averback, P. 1981. Parasynaptic corpora amylacea in the striatum. Arch. Pathol. Lab. Med. 105, 334-335.

Barrios, F.A., Gonzalez, L., Favila, R., Alonso, M.E., Salgado, P.M., Diaz, R., FernandezRuiz, J., 2007. Olfaction and neurodegeneration in HD. Neuroreport 18, 73-76.

Braak, H., Alafuzoff, I., Arzberger, T., Kretzschmar, H., Del Tredici, K., 2006. Staging of Alzheimer disease-associated neurofibrillary pathology using paraffin sections and immunocytochemistry. Acta Neuropathol. 112, 389-404.

Braak, H., Braak, E., Bohl, J., 1993. Staging of Alzheimer-related cortical destruction. Eur. Neurol. 33, 403-408.

Braak, H., Braak, E., Bohl, J., Reintjes, R., 1996. Age, neurofibrillary changes, A $\beta-$ amyloid and the onset of Alzheimer's disease. Neurosci. Lett. 210, 87-90.

Brai, E., Alberi, L., 2018. Olfaction, among the first senses to develop and decline. In: Sensory Nervous System, Chapter 4. Intechopen. pp. 65.

Brai, E., Alberi, L., 2015. Simple and computer-assisted olfactory testing for mice. J. Vis. Exp. e52944.

Brai, E., Alina Raio, N., Alberi, L., 2016. Notch1 hallmarks fibrillary depositions in sporadic Alzheimer's disease. Acta Neuropathol. Commun. 4, 64.

Brai, E., Marathe, S., Astori, S., Fredj, N.B., Perry, E., Lamy, C., Scotti, A., Alberi, L., 2015. Notch1 regulates hippocampal plasticity through interaction with the reelin pathway, glutamatergic transmission and CREB signaling. Front. Cell. Neurosci. 9, 447.

Brai, E., Marathe, S., Zentilin, L., Giacca, M., Nimpf, J., Kretz, R., Scotti, A., Alberi, L., 2014. Notch 1 activity in the olfactory bulb is odour-dependent and contributes to olfactory behaviour. Eur. J. Neurosci. 40, 3436-3449.

Cavanagh, J.B., 1998. Spinal corpora amylacea and motor neuron disease: a quantitative study. J. Neurol. Neurosurg. Psychiatry 65, 488-491.

Cheng, X., He, P., Lee, T., Yao, H., Li, R., Shen, Y., 2014. High activities of BACE1 in brains with mild cognitive impairment. Am. J. Pathol. 184, 141-147.

Chin, J., Massaro, C.M., Palop, J.J., Thwin, M.T., Yu, G.-O., Bien-Ly, N., Bender, A Mucke, L., 2007. Reelin depletion in the entorhinal cortex of human amyloid precursor protein transgenic mice and humans with Alzheimer's disease. J. Neurosci. 27, 2727-2733.

Cuchillo-Ibañez, I., Mata-Balaguer, T., Balmaceda, V., Arranz, J.J., Nimpf, J., SáezValero, J., 2016. The $\beta$-amyloid peptide compromises Reelin signaling in Alzheimer's disease. Sci. Rep. 6, 31646.

Davies, D.C., Brooks, J.W., Lewis, D.A., 1993. Axonal loss from the olfactory tracts in Alzheimer's disease. Neurobiol. Aging 14, 353-357.

Day, R.J., Mason, M.J., Thomas, C., Poon, W.W., Rohn, T.T., 2015. Caspase-cleaved tau co-localizes with early tangle markers in the human vascular dementia brain. PLoS One 10, e0132637.

Devanand, D.P., Lee, S., Manly, J., Andrews, H., Schupf, N., Doty, R.L., Stern, Y., Zahodne, L.B., Louis, E.D., Mayeux, R., 2014. Olfactory deficits predict cognitive decline and Alzheimer dementia in an urban community. Neurology 84, $182-189$.

Doehner, J., Genoud, C., Imhof, C., Krstic, D., Knuesel, I., 2012. Extrusion of misfolded and aggregated proteins-a protective strategy of aging neurons? Eur. J. Neurosci. 35, 1938-1950.

Doehner, J., Madhusudan, A., Konietzko, U., Fritschy, J.-M., Knuesel, I., 2010. Colocalization of Reelin and proteolytic AßPP fragments in hippocampal plaques in aged wild-type mice. J. Alzheimers. Dis. 19, 1339-1357.

Doty, R.L., Kamath, V., 2014. The influences of age on olfaction: a review. Front Psychol. 5, 20.

Galli, R., Meinhardt, M., Koch, E, Schackert, G., Steiner, G., Kirsch, M., Uckermann, O., 2018. Optical molecular imaging of corpora amylacea in human brain tissue. Biomed. Tech. (Berl) 63, 579-585.

Giridhar, S., Doiron, B., Urban, N.N., 2011. Timescale-dependent shaping of correlation by olfactory bulb lateral inhibition. Proc. Natl. Acad. Sci. U S A 108, 5843-5848.
Gouras, G.K., Almeida, C.G., Takahashi, R.H., 2005. Intraneuronal Abeta accumulation and origin of plaques in Alzheimer's disease. Neurobiol. Aging 26, 1235-1244. Hemming, M.L., Elias, J.E., Gygi, S.P., Selkoe, D.J., 2009. Identification of betasecretase (BACE1) substrates using quantitative proteomics. PLoS One 4, e8477.

Herring, A., Donath, A., Steiner, K.M., Widera, M.P., Hamzehian, S., Kanakis, D., Kölble, K., ElAli, A., Hermann, D.M., Paulus, W., Keyvani, K., 2012. Reelin depletion is an early phenomenon of Alzheimer's pathology. J. Alzheimers. Dis. 30, 963-979.

Herrmann, G., Mishev, G., Scotti, A.L., 2008. Olfactory bulb interneurons releasing NO exhibit the Reelin receptor ApoEr2 and part of those targeted by NO express Reelin. J. Chem. Neuroanat. 36, 160-169.

Herz, J., Chen, Y., 2006. Reelin, lipoprotein receptors and synaptic plasticity. Nat. Rev. Neurosci. 7, 850-859.

Hock, C., Golombowski, S., Müller-Spahn, F., Peschel, O., Riederer, A., Probst, A., Mandelkow, E., Unger, J., 1998. Histological markers in nasal mucosa of patients with Alzheimer's disease. Eur. Neurol. 40, 31-36.

Hüttenbrink, K.-B., Hummel, T., Berg, D., Gasser, T., Hähner, A., 2013. Olfactory dysfunction: common in later life and early warning of neurodegenerative disease. Dtsch. Arztebl. Int. 110, e1.

Ineichen, B.V., Weinmann, O., Good, N., Plattner, P.S., Wicki, C., Rushing, E.J., Linnebank, M., Schwab, M.E., 2017. Sudan black: a fast, easy and non-toxic method to assess myelin repair in demyelinating diseases. Neuropathol. Appl. Neurobiol. 43, 242-251.

Knuesel, I., Nyffeler, M., Mormède, C., Muhia, M., Meyer, U., Pietropaolo, S., Yee, B.K., Pryce, C.R., LaFerla, F.M., Marighetto, A., Feldon, J., 2009. Age-related accumulation of Reelin in amyloid-like deposits. Neurobiol. Aging 30, 697-716.

Kohl, Z., Schlachetzki, J.C.M., Feldewerth, J., Hornauer, P., Münch, M., Adame, A. Riemenschneider, M.J., Winkler, J., Masliah, E., 2017. Distinct pattern of microgliosis in the olfactory bulb of neurodegenerative proteinopathies. Neural Plast. 2017, 3851262.

Kovacs, G.G., Klöppel, S., Fischer, I., Dorner, S., Lindeck-Pozza, E., Birner, P., Bötefür, I.C., Pilz, P., Volk, B., Budka, H., 2003. Nucleus-specific alteration of raphe neurons in human neurodegenerative disorders. Neuroreport 14, 73-76.

Kovács, T., 2004. Mechanisms of olfactory dysfunction in aging and neurodegenerative disorders. Ageing Res. Rev. 3, 215-232.

Kubota, T., Naumann, G.O.H., 1993. Reduction in number of corpora amylacea with advancing histological changes of glaucoma. Graefes Arch. Clin. Exp. Ophthalmol. 231, 249-253.

Kulijewicz-Nawrot, M., Verkhratsky, A., Chvátal, A., Syková, E., Rodríguez, J.J., 2012. Astrocytic cytoskeletal atrophy in the medial prefrontal cortex of a triple transgenic mouse model of Alzheimer's disease. J. Anat. 221, 252-262.

LaFerla, F.M., Green, K.N., Oddo, S., 2007. Intracellular amyloid- $\beta$ in Alzheimer's disease. Nat. Rev. Neurosci. 8, 499-509.

Larson, J., Hoffman, J.S., Guidotti, A., Costa, E., 2003. Olfactory discrimination learning deficit in heterozygous reeler mice. Brain Res. 971, 40-46.

Liu, L., Drouet, V., Wu, J.W., Witter, M.P., Small, S.A., Clelland, C., Duff, K., 2012. Transsynaptic spread of tau pathology in vivo. PLoS One 7, e31302.

Li, W., Howard, J.D., Gottfried, J.A., 2010. Disruption of odour quality coding in piriform cortex mediates olfactory deficits in Alzheimer's disease. Brain 133, 2714-2726.

Loeffler, K.U., Edward, D.P., Tso, M.O., 1993. Tau-2 immunoreactivity of corpora amylacea in the human retina and optic nerve. Invest. Ophthalmol. Vis. Sci. 34, 2600-2603.

Marathe, S., Jaquet, M., Annoni, J.-M., Alberi, L., 2017. Jagged1 is altered in Alzheimer's disease and regulates spatial memory processing. Front. Cell. Neurosci. $11,220$.

Martin, J.E., Mather, K., Swash, M., Garofalo, O., Leigh, P.N., Anderton, B.H., 1991. Heat shock protein expression in corpora amylacea in the central nervous system: clues to their origin. Neuropathol. Appl. Neurobiol. 17, 113-119.

Mathur, R., Ince, P.G., Minett, T., Garwood, C.J., Shaw, P.J., Matthews, F.E., Brayne, C., Simpson, J.E., Wharton, S.B., MRC Cognitive Function and Ageing Neuropathology Study Group, 2015. A reduced astrocyte response to $\beta$-amyloid plaques in the ageing brain associates with cognitive impairment. PLoS One 10, e0118463.

Mesholam, R.I., Moberg, P.J., Mahr, R.N., Doty, R.L., 1998. Olfaction in neurodegenerative disease. Arch. Neurol. 55, 84.

Mundiñano, I.-C., Caballero, M.-C., Ordóñez, C., Hernandez, M., DiCaudo, C., Marcilla, I., Erro, M.-E., Tuñon, M.-T., Luquin, M.-R., 2011. Increased dopaminergic cells and protein aggregates in the olfactory bulb of patients with neurodegenerative disorders. Acta Neuropathol. 122, 61-74.

Nehring, L.C., Miyamoto, A., Hein, P.W., Weinmaster, G., Michael Shipley, J., 2005. The extracellular matrix protein MAGP-2 interacts with Jagged 1 and induces its shedding from the cell surface. J. Biol. Chem. 280, 20349-20355.

Notter, T., Knuesel, I., 2013. Reelin immunoreactivity in neuritic varicosities in the human hippocampal formation of non-demented subjects and Alzheimer's disease patients. Acta Neuropathol. Commun. 1, 27.

Okuyama-Yamamoto, A., Yamamoto, T., Miki, A., Terashima, T., 2005. Changes in reelin expression in the mouse olfactory bulb after chemical lesion to the olfactory epithelium. Eur. J. Neurosci. 21, 2586-2592.

Osborn, L.M., Kamphuis, W., Wadman, W.J., Hol, E.M., 2016. Astrogliosis: an integral player in the pathogenesis of Alzheimer's disease. Prog. Neurobiol. 144, 121-141.

Pirici, D., Margaritescu, C., 2014. Corpora amylacea in aging brain and age-related brain disorders. J. Aging Gerontol. 2, 33-57. 
Pirici, I., Mărgăritescu, C., Mogoantă, L., Petrescu, F., Simionescu, C.E., Popescu, E.S., Cecoltan, S., Pirici, D., 2014. Corpora amylacea in the brain form highly branched three-dimensional lattices. Rom. J. Morphol. Embryol. 55, 1071-1077.

Pisa, D., Alonso, R., Marina, A.I., Rábano, A., Carrasco, L., 2018. Human and microbial proteins from corpora amylacea of Alzheimer's disease. Sci. Rep. 8, 9880.

Risacher, S.L., Tallman, E.F., West, J.D., Yoder, K.K., Hutchins, G.D., Fletcher, J.W., Gao, S., Kareken, D.A., Farlow, M.R., Apostolova, L.G., Saykin, A.J., 2017. Olfactory identification in subjective cognitive decline and mild cognitive impairment: association with tau but not amyloid positron emission tomography. Alzheimers. Dement. 9, 57-66.

Rohn, T.T., 2015. Corpora amylacea in neurodegenerative diseases: cause or effect? Int. J. Neurol. Neurother 2, 031.

Roux, L., Benchenane, K., Rothstein, J.D., Bonvento, G., Giaume, C., 2011. Plasticity of astroglial networks in olfactory glomeruli. Proc. Natl. Acad. Sci. U S A 108 , $18442-18446$.

Sáez-Valero, J., Costell, M., Sjögren, M., Andreasen, N., Blennow, K., Luque, J.M., 2003. Altered levels of cerebrospinal fluid reelin in frontotemporal dementia and Alzheimer's disease. J. Neurosci. Res. 72, 132-136.

Saiz-Sanchez, D., Flores-Cuadrado, A., Ubeda-Bañon, I., de la Rosa-Prieto, C., Martinez-Marcos, A., 2016. Interneurons in the human olfactory system in Alzheimer's disease. Exp. Neurol. 276, 13-21.

Saiz-Sanchez, D., Ubeda-Bañon, I., de la Rosa-Prieto, C., Argandoña-Palacios, L., Garcia-Muñozguren, S., Insausti, R., Martinez-Marcos, A., 2010. Somatostatin, tau, and beta-amyloid within the anterior olfactory nucleus in Alzheimer disease. Exp. Neurol. 223, 347-350.

Sattler, R., Ayukawa, Y., Coddington, L., Sawa, A., Block, D., Chipkin, R., Rothstein, J.D., 2011. Human nasal olfactory epithelium as a dynamic marker for CNS therapy development. Exp. Neurol. 232, 203-211.

Schoppa, N.E., 2006. Synchronization of olfactory bulb mitral cells by precisely timed inhibitory inputs. Neuron 49, 271-283.

Cervello, A., Fioretti, A., Gualdi, G., Di Biasi, C., Pittalis, A., Sollaku, S., Pavaci, S., Tortorella, F, Fusetti, M., Valenti, M., Masedu, F, Cacciafesta, M., Marigliano, V. Ettorre, E., Pagliarella, M., 2015. Olfactory dysfunction, olfactory bulb volume and Alzheimer's disease: is there a correlation? A pilot study1. J. Alzheimers. Dis. $48,395-402$.

Singhrao, S.K., Morgan, B.P., Neal, J.W., Newman, G.R., 1995. A functional role for corpora amylacea based on evidence from complement studies. Neurodegeneration 4, 335-345.

Tate-Ostroff, B., Majocha, R.E., Marotta, C.A., 1989. Identification of cellular and extracellular sites of amyloid precursor protein extracytoplasmic domain in normal and Alzheimer disease brains. Proc. Natl. Acad. Sci. U S A 86, 745-749.

ter Laak, H. Renkawek, K, van Workum, F.P. 1994. The olfactory bulb in Alzheimer disease: a morphologic study of neuron loss, tangles, and senile plaques in relation to olfaction. Alzheimer Dis. Assoc. Disord. 8, 38-48.

Thomann, P.A., Dos Santos, V., Seidl, U., Toro, P., Essig, M., Schröder, J., 2009. MRIderived atrophy of the olfactory bulb and tract in mild cognitive impairment and Alzheimer's disease. J. Alzheimers. Dis. 17, 213-221.

Trotter, J., Lee, G.H., Kazdoba, T.M., Crowell, B., Domogauer, J., Mahoney, H.M., Franco, S.J., Muller, U., Weeber, E.J., D’Arcangelo, G., 2013. Dab1 is required for synaptic plasticity and associative learning. J. Neurosci. 33, 15652-15668.

Wang, Y., Chan, S.L., Miele, L., Yao, P.J., Mackes, J., Ingram, D.K., Mattson, M.P. Furukawa, K., 2004. Involvement of Notch signaling in hippocampal synaptic plasticity. Proc. Natl. Acad. Sci. U S A 101, 9458-9462.

Weeber, E.J., Beffert, U., Jones, C., Christian, J.M., Forster, E., Sweatt, J.D., Herz, J., 2002. Reelin and ApoE receptors cooperate to enhance hippocampal synaptic plasticity and learning. J. Biol. Chem. 277, 39944-39952.

Wesson, D.W. Levy, E., Nixon, R.A., Wilson, D.A., 2010. Olfactory dysfunction correlates with amyloid- burden in an Alzheimer's disease mouse model. J. Neurosci. 30, 505-514.

Yao, Z.-G., Hua, F., Zhang, H.-Z., Li, Y.-Y., Qin, Y.-J., 2017. Olfactory dysfunction in the APP/PS1 transgenic mouse model of Alzheimer's disease: morphological evaluations from the nose to the brain. Neuropathology 37, 485-494.

Yoo, S.-J., Lee, J.-H., Kim, S.Y., Son, G., Kim, J.Y., Cho, B., Yu, S.-W., Chang, K.-A., Suh, Y.H., Moon, C., 2017. Differential spatial expression of peripheral olfactory neuronderived BACE1 induces olfactory impairment by region-specific accumulation of $\beta$-amyloid oligomer. Cell Death Dis. 8, e2977. 